\title{
Qualitative analysis of the response regimes and triggering mechanism of bistable NES
}

\author{
Zhenhang Wu • Sébastien Seguy • \\ Manuel Paredes
}

Received: date / Accepted: date

\begin{abstract}
The main focus of this study is the development of an adapted complex variable method in the vicinity of equilibrium in bistable NES. A simplified chaos trigger model is established to describe the distance between the stable phase cycle and the pseudo-separatrix. An analytical expression can predict the excitation threshold for chaos occurrence. The relative positions between the chaos trigger threshold line and the Slow Invariant Manifold (SIM) structure can express the distribution of response regimes under growing harmonic excitation. This topological structure implies the alternation of the response regime and helps to classify the bistable NES. The experiment compares the analytical result of intra-well oscillation with the numerical result in the frequency domain. The experimental response regimes under different input energy levels and frequency domains have been observed and give ideas to guide the optimal design of a bistable NES. It is shown that the modest bistable NES possesses strong robustness to frequency perturbation.
\end{abstract}

Keywords Qualitative analysis · Bistable nonlinear energy sink · Slow invariant manifold · Targeted energy transfer · Trigger mechanism

Declarations The authors have no conflicts of interest to declare that are relevant to the content of this article. The authors declare that the data supporting the findings of this study are available within the article. The authors declare that all procedures performed in this study were in accordance with the

Zhenhang Wu

Institut Clément Ader (ICA), CNRS, INSA-ISAE-Mines Albi-UPS, Université de Toulouse,

3 rue Caroline Aigle, Toulouse, 31400, France

Tel.: +0761919078

E-mail: zhenhang.wu@insa-toulouse.fr

Sébastien Seguy

sebastien.seguy@insa-toulouse.fr

Manuel Paredes

manuel.paredes@insa-toulouse.fr 
ethical standards of COPE. This manuscript is only submitted to the journal 'Nonlinear Dynamics'. The authors declare that this study is complete, unsplit and original.

Acknowledgments The authors acknowledge the Chinese Scholarship Council under Grant No. 201801810128 for their financial support.

\section{Introduction}

The Nonlinear Energy Sink (NES), a novel vibration absorber, has become an active research field in recent decades. It consists of nonlinear stiffness and viscous damping connecting the primary mass and NES mass. The traditional Tuned Mass Damper (TMD) has to be tuned to closely match the mechanical system's natural frequency in order to absorb more energy. The performance of a TMD will decline dramatically if the applied excitation frequency shifts. In contrast, the NES possesses a broader range of absorbed frequencies because of its self-adaptive stiffness, and its energy-dependent resonating frequency characteristic [1]. The NES also has some other remarkable advantages: a lighter attached mass, and a capability to wipe out the resonance peak [2].

The most significant properties are that the energy of the Linear Oscillator (LO) is irreversibly transferred into the NES and is rapidly dissipated by the damping [3]. This phenomenon, called Targeted Energy Transfer (TET), is based on 1:1 resonance capture. If the primary system is under harmonic forcing, it gives rise to a beating response, which is referred to as a Strongly Modulated Response (SMR). The appearance of folded singularities in the Slow Invariant Manifold (SIM) implies a necessary excitation threshold condition to activate the SMR [4]. The stability of the SMR is transformed into a 1-D mapping problem. The analytical approach provides a necessary damping condition to ensure SMR [5]. The NES concept has been explored in both numerical $[6,7]$ and experimental ways $[8,9]$.

Various types of NES, such as piecewise NES [10], rotary NES [11], and vibro-impact NES (VI NES) [12,25], have been investigated to better explore the potential of NES. The absorption performance of different configurations among cubic NES and bistable NES shows the priority of the latter NES to reduce the band width of the initial energies input $[14,15]$. Single-sided VI NES leading to more effective shock mitigation are compared to a doublesided restricted VI NES [16]. The mechanical applications in structural seismic control [17] and in mitigating chatter vibration [18] in the tuning process have been studied.

Nowadays, one of the widely used analytical approaches for processing NES is the complexification averaging ( $\mathrm{CX}-\mathrm{A})$ method to derive the modulation equation and compute the fixed points [19]. The application of the standard multiple-scales procedure gives a slow/fast partition of the dynamics by introducing a fast time scale $\tau$ and a slow timescale $\tau_{1}=\epsilon \tau[2-7]$. However, as for bistable NES, which involves essential chaotic motion, a rigorous theoretical description is not possible. The analytical study presented here provides an 
adequate description of the initial highly energetic regime of intensive energy transfer from LO to NES on a reduced system without considering damping and excitation [20]. A study of parameters, based on an approach complexifying the Hamiltonian system, reveals the frequency-energy characteristic of the bistable case. The backbone of periodic solutions of the conservative system in the frequency-energy plane depicts in-phase (S11+) and out-of-phase (S11-) 1:1 resonance oscillations [21], which are responsible for the intensive energy exchange in Hamiltonian systems.

The classification of bistable NES response regimes is mainly based on empirical observation. In a low energy case, subharmonic resonances and chaotic cross-well oscillations are excited. In a higher energy case, the fundamental (1:1) and subharmonic (1:3) resonances mainly govern the dynamic behaviour. In [22], an extension of Manevitch's complex variables shows that it is potentially better to describe higher harmonics in an initial high energy input. If the bistable NES system is under harmonic excitation input, four typical response regimes at different energy levels appear in turn: (1) intra-well oscillation, (2) chaotic inter-well oscillation, (3) Strongly modulated response, (4) steadystate [23]. The optimal point occurs in the transition from the SMR stage to a stable response. Adjusting a variable pitch spring can provide the desired nonlinear stiffness with the optimal design [25]. The robustness of optimal design is verified in [16], which also concludes that the damping condition mainly determines the ceiling of maximum efficiency in the optimal cubic NES.

To construct and extend flexible use of bistable NES in different mechanical contexts, several materials have been tested, e.g., cantilever beam [26], magnetic material $[27,28]$, bistable thin plate [29], spring system [23,30], and buckled beam[31].

This work is organised as follows. In Section 2, an adapted complex variable is developed to predict the excitation threshold for chaos to occur. Section 3 proposes a simplified trigger chaos model and verifies it numerically. In Section 4, the relative position of the chaos trigger line and its SIM structure express an alternating phenomenon of response regimes and, in Section 5, the experimental result verifies the analytical intra-well result calculated using the adapted complex variables method. Section 6 investigates the frequency distribution of three negative stiffness cases from the previous section. The last section mentions some noteworthy conclusions.

\section{Adapted complex variables method}

Intra-well oscillation relates to a low energy motion that is restricted to one of the potential wells. It will become chaotic with increasing energy. An exact method to describe the intra-well oscillation is necessary to divide the regimes. There are currently two effective methods for identifying potential chaos oscillation. Firstly, the Lyapunov exponents (LEs) measure the degree of stochasticity of a trajectory by defining a relative distance in phase space between a given orbit and a test orbit beginning with a nearby initial condi- 


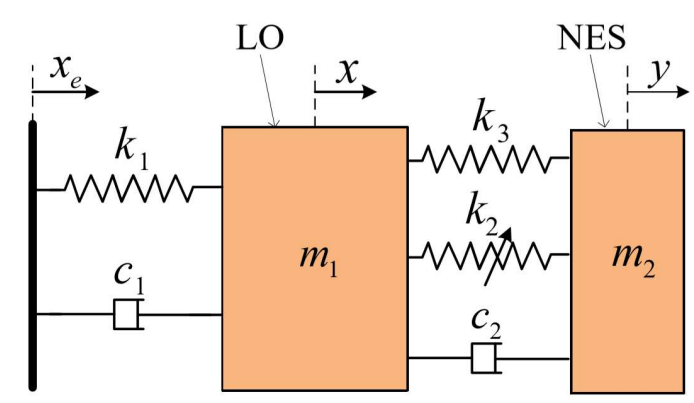

Fig. 1 The schematic of bistable NES system

tion. An LE approaching a positive value implies a chaotic orbit [32]. However, the LEs are difficult to calculate analytically. The numerical calculation is also restricted by an infinitesimal initial distance and an infinite time horizon. So a practical Wolf's algorithm is applied in $[32,33]$. The Melnikov method is another of the few analytical ways to obtain an approximate criterion for chaos and homoclinic bifurcation. According to the Melnikov theory, the chaos and transverse interaction over the separatrix in the phase plane occur simultaneously. The threshold values of damping and other control parameters for the occurrence of homoclinic bifurcation are presented in [16,34]. Both ways can hardly predict the force threshold for the occurrence of chaos under harmonic excitation.

A definition of the amplitude of the NES is necessary to predict the threshold force for chaotic motion. The traditional treatment of $w$ defines it as the relative distance between LO and NES. However, the negative stiffness generates one equilibrium on either side of the origin of the coordinates. The small oscillation around equilibria will be described as a large amplitude. The phase trajectory at a low energy level will not fit alongside a SIM structure. It also generates a massive error in the analytical calculation of NES amplitude. So it is necessary to consider the position of equilibrium and define the distance between the NES and the equilibrium point as a relative displacement.

First of all, the target system consists of a linear oscillator (LO) $m_{1}$, which is sustained by a harmonic excitation $x_{e}=G \cos (\omega t)$ through linear stiffness $k_{1}$ and viscous damping coefficient $c_{1}$. A lightweight $m_{2}$ is coupled to LO with viscous damping $c_{2}$ by means of cubic stiffness $k_{3}$ and negative stiffness $k_{2}$. The schema of a bistable NES system is presented in Fig. 1.

$$
\begin{aligned}
& m_{1} \ddot{x}+k_{1} x+c_{1} \dot{x}+c_{2}(\dot{x}-\dot{y})+k_{2}(x-y)^{3}+k_{3}(x-y)=k_{1} x_{e}+c_{1} \dot{x}_{e} \\
& m_{2} \ddot{y}+c_{2}(\dot{y}-\dot{x})+k_{2}(y-x)^{3}+k_{3}(y-x)=0
\end{aligned}
$$

The governing equation (1) can be written in rescaled form (3) by introducing the following rescaled variables (2). The new variable $v=x+\epsilon y$ represents 
the displacement of mass and $w=x-y$ is the relative displacement of the bistable NES.

$$
\begin{gathered}
\epsilon=\frac{m_{2}}{m_{1}}, \omega_{0}^{2}=\frac{k_{1}}{m_{1}}, K=\frac{k_{2}}{m_{2} \omega_{0}^{2}}, \delta=\frac{k_{3}}{m_{2} \omega_{0}^{2}} \\
\lambda_{1}=\frac{c_{1}}{m_{2} \omega_{0}}, \lambda_{2}=\frac{c_{2}}{m_{2} \omega_{0}}, F=\frac{G}{\epsilon}, \Omega=\frac{\omega}{\omega_{0}}, \tau=\omega_{0} t \\
\ddot{v}+\epsilon \lambda_{1} \frac{\dot{v}+\epsilon \dot{w}}{1+\epsilon}+\frac{v+\epsilon w}{1+\epsilon}=\epsilon F \cos \Omega \tau \\
\ddot{w}+\epsilon \lambda_{1} \frac{\dot{v}+\epsilon \dot{w}}{1+\epsilon}+\frac{v+\epsilon w}{1+\epsilon}+\lambda_{2}(1+\epsilon) \dot{w}+K(1+\epsilon) w^{3}+\delta(1+\epsilon) w=\epsilon F \cos \Omega t
\end{gathered}
$$

The system is investigated in the vicinity of $1: 1$ resonance where LO oscillates at the identical frequency $\Omega$. Two adapted complex variables describing the neighbourhood of positive stable equilibrium point $x_{0}=\sqrt{-\delta / K}$ are given by

$$
\begin{aligned}
& \phi_{1}(\tau) e^{i \Omega \tau}=\frac{d}{d \tau} v(\tau)+i \Omega\left(v(\tau)+\epsilon x_{0}\right) \\
& \phi_{2}(\tau) e^{i \Omega \tau}=\frac{d}{d \tau} w(\tau)+i \Omega\left(w(\tau)-x_{0}\right)
\end{aligned}
$$

with $i=\sqrt{-1}$ the imaginary unit. A minus sign should be added in (4) in order to study the local dynamics near the negative stable equilibrium $-x_{0}$. Only intra-well oscillation on the positive side falls within the scope of our present considerations, for the sake of symmetry.

In a low energy intra-well oscillation case, the NES vibrates symmetrically near the positive equilibrium as a centre. However, the numerical simulation confirms that the amplitude of LO is also slightly asymmetrical. The centre of LO oscillation approximately locates $-\epsilon x_{0}$. The initial adapted complex variables assumption best fits the simulation result.

Introducing (4) into (3), and keeping only terms containing $e^{i \Omega \tau}$ yields the following slow modulated system:

$$
\begin{aligned}
& \dot{\phi}_{1}+\frac{i \Omega}{2} \phi_{1}+\frac{\epsilon \lambda_{1}\left(\phi_{1}+\epsilon \phi_{2}\right)}{2(1+\epsilon)}-\frac{i\left(\phi_{1}+\epsilon \phi_{2}\right)}{2 \Omega(1+\epsilon)}-\frac{\epsilon F}{2}=0 \\
& \dot{\phi}_{2}+\frac{i \Omega}{2} \phi_{2}+\frac{\epsilon \lambda_{1}\left(\phi_{1}+\epsilon \phi_{2}\right)}{2(1+\epsilon)}-\frac{i\left(\phi_{1}+\varepsilon \phi_{2}\right)}{2 \Omega(1+\epsilon)}+\frac{\lambda_{2}(1+\epsilon) \phi_{2}}{2}-\frac{3 i K(1+\epsilon) \phi_{2}^{2} \bar{\phi}_{2}}{8 \Omega^{3}} \\
& -\frac{\epsilon F}{2}-\frac{i \phi_{2} \delta(1+\epsilon)}{2 \Omega}-\frac{3 i K(1+\epsilon) \phi_{2} x_{0}{ }^{2}}{2 \Omega}=0
\end{aligned}
$$

The stable response of intra-well oscillation corresponds to the fixed point of (5) when the derivative equals zero. Through an algebraic operation, the analytical amplitude of the system can be expressed in a more convenient form. Coefficients $\alpha_{i}(\mathrm{i}=1 . .3)$ are not given here due to their length.

$$
\begin{gathered}
\phi_{10}=\frac{\frac{i \epsilon \phi_{20}}{\Omega(1+\varepsilon)}-\frac{\varepsilon^{2} \lambda_{1} \phi_{20}}{1+\varepsilon}+\varepsilon F+i \varepsilon^{2} \lambda_{1} F \Omega}{i \Omega+\frac{\varepsilon \lambda_{1}}{1+\varepsilon}-\frac{i}{\Omega(1+\varepsilon)}} \\
\alpha_{3} Z_{20}^{3}+\alpha_{2} Z_{20}^{2}+\alpha_{1} Z_{20}+\alpha_{0} F^{2}=0, \quad Z_{20}=\left|\phi_{20}\right|^{2}
\end{gathered}
$$

The stability of intra-well oscillation is studied by introducing a small perturbation into the fixed point equation (5).

$$
\phi_{1}=\phi_{10}+\rho_{1}, \quad \phi_{2}=\phi_{20}+\rho_{2}, \overline{\phi_{1}}=\overline{\phi_{10}}+\overline{\rho_{1}}, \overline{\phi_{2}}=\overline{\phi_{20}}+\overline{\rho_{2}}
$$




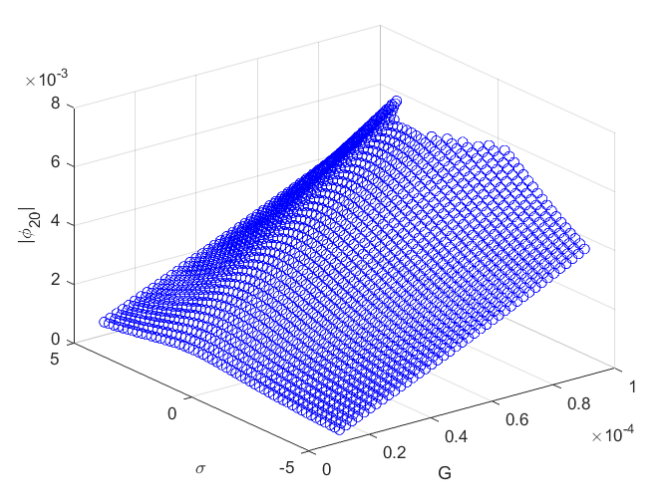

Fig. 2 Amplitude of intra well oscillation in the frequency domain with system parameters: $\epsilon=0.01, \lambda_{1}=1.67, \lambda_{2}=0.167, \mathrm{~K}=1743, \delta=-0.43$. Blue points indicate the stability of the solution.

Extracting the perturbation terms gives the characteristic matrix.

$$
\left[\begin{array}{c}
\dot{\rho}_{1} \\
\dot{\rho}_{2} \\
\dot{\rho}_{1} \\
\dot{\rho}_{2}
\end{array}\right]=\left[\begin{array}{cccc}
M_{11} & \epsilon M_{21} & 0 & 0 \\
M_{21} & M_{22} & 0 & M_{24} \\
0 & 0 & \bar{M}_{11} & \epsilon \bar{M}_{21} \\
0 & \bar{M}_{24} & \bar{M}_{21} & \bar{M}_{22}
\end{array}\right]\left[\begin{array}{c}
\rho_{1} \\
\rho_{2} \\
\bar{\rho}_{1} \\
\bar{\rho}_{2}
\end{array}\right]
$$

where

$$
\left\{\begin{array}{l}
M_{11}=-\frac{i(1+\epsilon)}{2}-\frac{\epsilon \lambda_{1}}{2(1+\epsilon)}+\frac{i}{2(1+\epsilon)(1+\epsilon \sigma)} \\
M_{21}=-\frac{\epsilon \lambda_{1}}{2(1+\epsilon)}+\frac{i}{2(1+\epsilon)(1+\epsilon \sigma)} \\
M_{22}=\frac{3 i(1+\epsilon) K \phi_{20} \bar{\phi}_{20}}{2(1+\epsilon)(1+\epsilon \sigma)}-\frac{\lambda_{2}(1+\epsilon)}{2}+\frac{i \epsilon}{2(1+\epsilon)(1+\epsilon \sigma)}-\frac{i(1+\epsilon \sigma)}{2}-\frac{\epsilon^{2} \lambda_{1}}{2(1+\epsilon)}+\frac{i(1+\epsilon)\left(3 K x_{0}^{2}+\delta\right)}{2(1+\epsilon \sigma)} \\
M_{24}=\frac{3 i(1+\epsilon) K \phi_{20}^{2}}{8(1+\epsilon \sigma)^{3}}
\end{array}\right.
$$

The small detuning parameter $\sigma$ is applied to measure how near the excitation frequency is to the natural frequency of LO. It gives $\Omega=1+\epsilon \sigma$. The existence of a root of the characteristic equation with a positive real part implies the instability of periodic intra-well oscillation, and vice versa.

The stability of local equilibrium oscillation is deduced and presented in Fig. 2 in the frequency domain. All blue points mean that all the real roots are located in the left-half complex plane. The motion within the well is naturally stable in our case. The absolute value of $\phi_{20}$ is lower than the chaos threshold. It ensures that the local dynamics is restricted to within the well. If the value of $\phi_{20}$ exceeds the chaos threshold, it is beyond the scope of our present section, and results in truncation in the vicinity of the natural frequency in Fig. 2. 
(a)

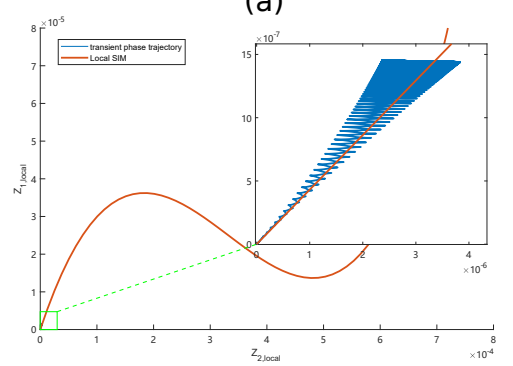

(b)

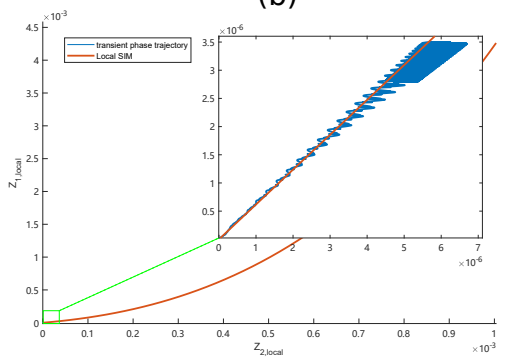

Fig. 3 SIM and local phase trajectory for $k_{3}=-20(\delta=-0.174$, left $), k_{3}=-100(\delta=$ -0.871 , right). Zoomed insert represents the detailed phase trajectory of intra-well oscillation defined by Equation 6 in the green frame

\subsection{Asymptotic analysis of local SIM}

When considering the adapted variables method, the SIM structure in which the classical multiple-scales method is applied has to be reconstructed.

$$
\begin{gathered}
\phi_{i}=\phi_{i}\left(\tau_{0}, \tau_{1}, \ldots\right), \frac{\mathrm{d}}{\mathrm{d} \tau}=\frac{\partial}{\partial \tau_{0}}+\epsilon \frac{\partial}{\partial \tau_{1}}+\epsilon^{2} \frac{\partial}{\partial \tau_{2}}+\cdots \\
\tau_{k}=\epsilon^{k} \tau, \quad k=0,1, \ldots
\end{gathered}
$$

The dynamic behaviour is considered to involve motion on various times scales. $\tau_{0}$ represents fast time scales, and $\tau_{1}=\epsilon \tau_{0}$ slow time scales. The rule for derivation under different time scales is presented in (10). By substituting (10) into (9), the terms involving the same power of $\epsilon$, are selected:

$$
\begin{gathered}
\frac{\partial}{\partial \tau_{0}} \phi_{1}=0 \\
\frac{\partial}{\partial \tau_{0}} \phi_{2}+\frac{1}{2} \lambda_{2} \phi_{2}+\frac{1}{2} i\left(\phi_{2}-\phi_{1}\right)-\frac{1}{2} i \delta \phi_{2}-\frac{3}{2} i K \phi_{2} x_{0}^{2}-\frac{3}{8} i K \bar{\phi}_{2} \phi_{2}^{2}=0
\end{gathered}
$$

where the first equation in (11) indicates that the modulation of $\phi_{1}$ is independent of $\tau_{0}$. Fixed point $\Phi=\lim _{\tau_{0} \rightarrow \infty} \phi_{2}$ obeys the algebraic equation:

$$
\frac{1}{2} \lambda_{2} \Phi+\frac{1}{2} i\left(\Phi-\phi_{1}\right)-\frac{1}{2} i \delta \Phi-\frac{3}{2} i K \Phi{x_{0}}^{2}-\frac{3}{8} i K \bar{\Phi} \Phi^{2}=0
$$

Taking $\Phi\left(\tau_{1}\right)=N_{2} e^{i \delta_{2}}$ and solving the above equation:

$$
Z_{1}=\left(\lambda_{2}^{2}+(\delta-1)\left(\delta-1+6 K x_{0}^{2}+\frac{3}{2} K Z_{2}\right)+9 K^{2}\left(x_{0}^{2}+\frac{Z_{2}}{4}\right)^{2}\right) Z_{2}
$$

where $Z_{1}=\left|\phi_{1}\right|^{2}, Z_{2}=|\Phi|^{2}$. An illustration of the modified SIM is given in Fig. 3 under the different negative stiffnesses where $\epsilon=0.01, \lambda_{1}=1.67, \lambda_{2}=$ $0.167, K=1742$.

In a cases of small negative stiffness, the global SIM possesses a characteristic similar to the classic cubic SIM curve, which has singular points like Fig. 3(a). Although most of the SIM curve is beyond the scope of this application, the zoomed insert part in the vicinity of point $(0,0)$ shows that the phase trajectory climbs along with the SIM. In a more significant negative stiffness 
case, in Fig. 3(b), the global SIM becomes a monotonically increasing curve. The phase trajectory of the zoomed insert part still oscillates around the SIM, which shows that it is correct for low energy input.

Unlike the traditional description method, e.g. case 1 in Fig. 8, where the phase trajectory has fully separated itself from the SIM, the local SIM describes intra-well motion more accurately. This modified SIM structure is developed based on the adapted variable method and its application scope is restricted to an intra-well oscillation. So, the SIM describes its dynamic behaviours with sufficient accuracy only in a low energy input case. A more significant energy input will result in its failure.

\subsection{Performance verification}

The validity of the adapted complex method can be verified by comparing it with numerical simulations. The difference between amplitudes of $w$ and $v$ calculated by (6), which is indicated by the red line with red dots at both ends above or below the surface, and direct numerical calculation (surface) is presented in Fig. 4 for the various negative stiffness cases $\left(k_{3}=-20\right.$ and $\left.k_{3}=-100\right)$ with system parameters: $\epsilon=0.01, \lambda_{1}=1.67, \lambda_{2}=0.167, \mathrm{~K}=1743$, $\delta=-0.43$. These parameters were kept constant in the following numerical simulation.

The adapted method generates errors mainly in the vicinity of $\sigma=0$ in Fig. 4. What can be observed is the growth of accuracy away from natural frequency. The amplitude of $\mathrm{w}$ and $\mathrm{v}$ steady rises with the augment of excitation G, resulting in NES oscillating in a more significant span and even finally exceeding the potential well. This kind of error can be understood by the dissatisfying of the initial assumption.

In a weak negative stiffness case, the analytical amplitude $w$ is always more significant than the numerical result as in Fig. 4(a). In a more intense $\delta$ case, the adapted complex method generates smaller amplitude w in Fig. 4(c), where the red lines with red dots is still below the amplitude surface. As for the accuracy of $\mathrm{v}$ calculation, Fig. 4(d) shows a larger analytical result. The amplitude of LO shows a good fit result compared with direct numerical simulation in Fig. 4(b). The absolute maximum error in both w calculation is $1.2 \%$ and $2.5 \%$ respectively, which is acceptable for our future study.

\section{Analytical prediction of chaotic motion}

\subsection{Simplified model for chaos occurrence}

Chaos always occurs in the transition from intra-well oscillation to inter-well oscillation. The Melnikov method is one of the few effective methods for finding the necessary condition for homoclinic bifurcation and predicting chaotic motion. According to [1], the unperturbed homoclinic orbit that connects the saddle points is given by: 
(a)

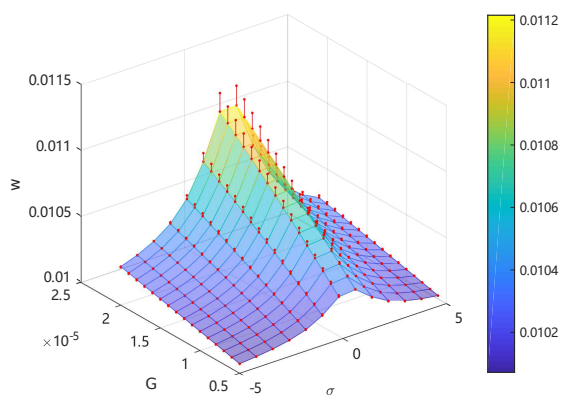

(c)

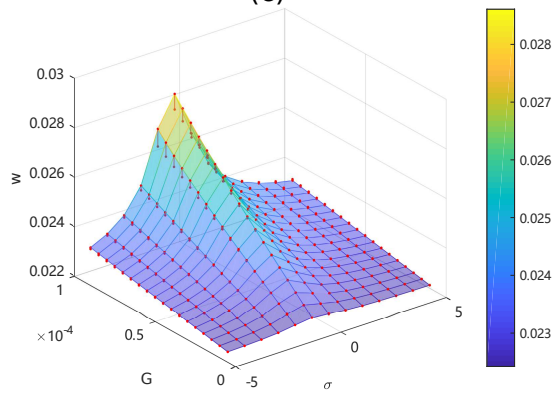

(b)

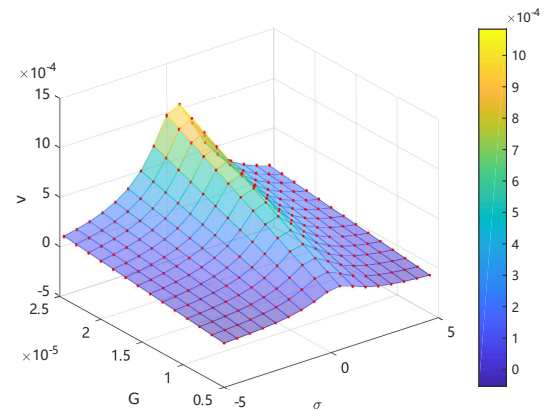

(d)

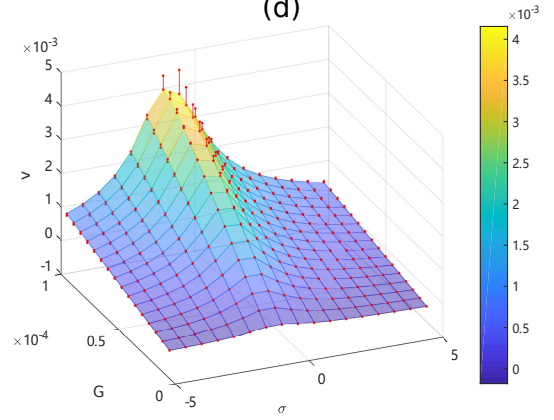

Fig. 4 Comparison of numerical calculation and analytical result of NES. (a), (b) are the displacement of NES and LO in a weak negative stiffness case, $k_{3}=-20$. (c), (d) are the displacement of NES and LO in a strong negative stiffness case, $k_{3}=-100$. The surface is the direct numerical result from the ODE45 function in Matlab. Red lines with red dots at both ends represent the difference between the analytical and numerical results

$$
\begin{aligned}
& q_{+}^{0}(\tau)=(R \cdot \operatorname{sech}(S \tau),-R S \cdot \operatorname{sech}(S \tau) \tanh (S \tau)) \\
& q_{-}^{0}(\tau)=-q_{+}^{0}(\tau)
\end{aligned}
$$

where $R=\sqrt{-\delta / K}, S=\sqrt{-\delta}$.

The NES oscillates around the attractor (equilibrium) with a small amplitude, and the circle can describe its corresponding stable phase trajectory with sufficient accuracy in the low energy input condition. The phase trajectory expands in a circle with the its centre at the attractor point $\left(x_{0}, 0\right)$. Its intersection with the pseudo-separatrix (red line in Fig. 5) can be considered as a symbol of the occurrence of chaos. In the different $\delta$ cases, the trigger condition is different as shown in Fig. 5. The critical $\delta$ value divides the trigger conditions into two types: (1) with the contact point located on the pseudoseparatrix or (2) with the contact point located on the extreme right of the pseudo-separatrix.

During the transition from intra-well oscillation to chaotic inter-well oscillation, the phase trajectory will cross the pseudo-separatrix. The trigger condition can be determined by calculating the minimum distance between the point on the pseudo-separatrix and the attractor. The minimal distance $D$ 

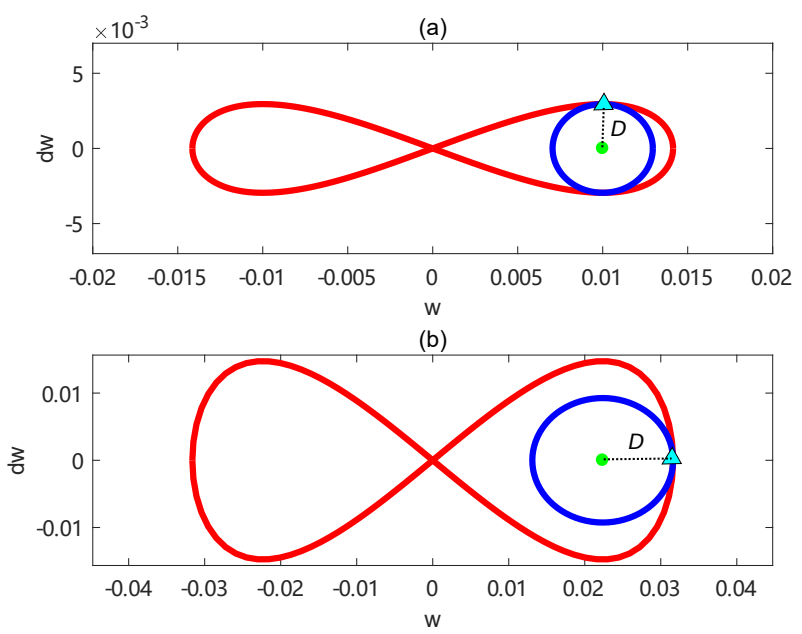

Fig. 5 Different trigger conditions in small $\delta$ value and strong $\delta$ value. The red line is the pseudo-separatrix, the blue line is the ideal phase trajectory, the green dot is the attractor centre $\left(x_{0}, 0\right)$, and the triangle is the contact point. Radius D gives the critical amplitude of NES for chaos to occur.

is the distance between contact point and attractor. The $D$ value, as a function of $w$, can be defined from (15):

$$
D^{2}=\left(w-\frac{1}{2} R \sqrt{2}\right)^{2}+\frac{S^{2} w^{2}\left(R^{2}-w^{2}\right)}{R^{2}}
$$

The local minimum distances, which exist only in the following three positions within the interval $[0, R]$, are obtained by taking the derivative of $w$ in the above equation and setting this derivative to zero.

$$
w_{1,2,3}=\frac{\left(-\frac{1}{4} \sqrt{2} S+\frac{1}{4} \sqrt{2 S^{2}+8}\right) R}{S}, \frac{R}{\sqrt{2}}, R
$$

If the negative stiffness $\delta$ exceeds the critical value $(2-\sqrt{2})^{2}$, (critical negative stiffness $k_{3}=-39.4$ in our case), the minimum distance is always equal to $\left(1-\frac{\sqrt{2}}{2}\right) R$, which means that the point on the pseudo-separatrix that is closest to the attractor point is always located at the extreme right point, as case (b) in Fig. 5 shows.

In a case of relatively greater negative stiffness, it is reasonable to consider the distance between the extreme right point and the attractor as the critical amplitude. If the final stable amplitude $\left|\phi_{20}\right|$ exceeds the critical amplitude $\left|\phi_{20 c}\right|=\left(1-\frac{\sqrt{2}}{2}\right) R$, chaotic behaviour results. And the amplitude threshold of NES for chaos occurrence, $Z_{a}=\left|\phi_{20 c}\right|^{2}$ can be expressed in (17).

$$
Z_{a}=\left\{\begin{array}{rr}
\left(1-\frac{\sqrt{2}}{2}\right)^{2} R^{2} & |\delta|>(2-\sqrt{2})^{2} \\
\frac{R^{2} S^{2}}{4} & |\delta| \leq(2-\sqrt{2})^{2}
\end{array}\right.
$$




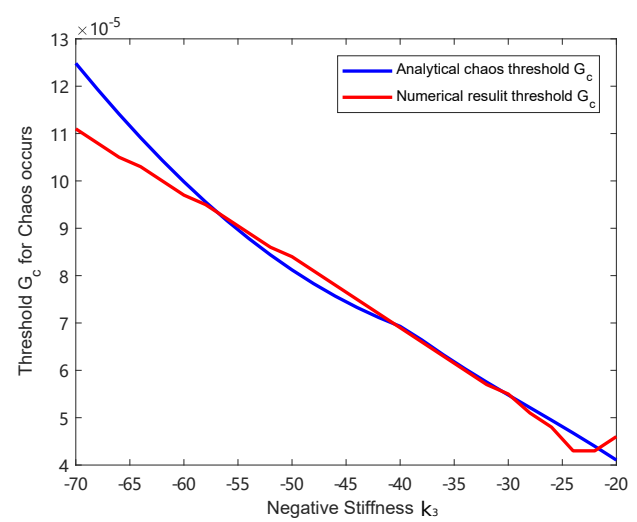

Fig. 6 Comparison of analytical chaos prediction with numerical result, with condition: $\epsilon$ $=0.01, \lambda_{1}=1.67, \lambda_{2}=0.167, \mathrm{~K}=1743, \sigma=0$

\subsection{Analytical chaos prediction}

The assumption is that the intra-well oscillation expands in a circle and intersects the pseudo-separatrix at critical amplitudes $\left|\phi_{20 c}\right|$ in various negative stiffness cases. If the system's amplitude increases monotonically before its phase trajectory crosses the pseudo separatrix, the trigger condition (17) can be substituted for the stable solution $Z_{20}$ in the second equation of (6). So the analytical excitation for chaos occurrence is as follows:

$$
G_{0 c}^{2}=-\frac{\epsilon Z_{20}\left(Z_{20}^{2} \alpha_{3}+Z_{20} \alpha_{2}+\alpha_{1}\right)}{\alpha_{0}}
$$

A more exact threshold value of excitation calculated by (18) can be compared with the numerical result in Fig. 6. The comparison reveals a gradual decline in excitation threshold for chaos occurrence as negative stiffness weakens. The more intense negative stiffness results in a deeper well requiring more energy input to escape from it, which leads to a higher excitation threshold.

The analytically predicted value is close to the numerical result in Fig. 6 . According to the performance verification section, the analytical amplitude of $w$ is smaller than the numerical amplitude in the strong negative case. This implies that the analytical amplitude of $w$ will give a more significant critical trigger excitation.

The accuracy of predictions is essentially changed in strong negative stiffness. The simplified model is less accurate if the phase trajectory is far away from the attractor. At the moment when a trajectory passes the pseudo separatrix, it is always at some distance from the attractor. This distance rises as the value of $\delta$ increases. So the analytical prediction of chaos is accurate only for cases of small negative stiffness.

From another point of view, the adapted complex variables method is based on the stable periodic solution triggering chaos. In a case of much higher 
negative stiffness, the instantaneous amplitude of the NES exceeds its final stable amplitude, which is inconsistent with the initial assumption that it is the final stable phase trajectory (final periodic solution), rather than the instantaneous amplitude, that triggers the pseudo-separatrix.

In addition, only one side attractor is under consideration; a greater negative stiffness makes the phase trajectory deviate from the ideal circle model. When the phase trajectory passes the mid point between the origin and the attractor, the other side attractor in the negative side will increase NES amplitude. The asymmetry of amplitude with respect to the attractor renders the prediction result invalid. In the vicinity of the pseudo-separatrix, the intra-well and inter-well subharmonic oscillations are beyond the descriptive capabilities of the adapted complex variables method. The above potential interpretation explains the generation of error in the process of application of the adapted complex variables method in the prediction of chaos.

\section{Qualitative analysis of response regimes}

The presentation of negative stiffness introduces chaotic motion essentially changing the response regimes. Two main characteristics are discussed below to interpret the response regimes in various negative stiffness cases.

First, the pseudo-separatrix governs the low energy behaviours and distinguishes them from chaotic motion. Low energy restricts the NES to oscillation around the attractor $(\sqrt{-\delta / K}, 0)$, and expands in one well with growing energy. If the amplitude of w exceeds the extreme right point $(\sqrt{-2 \delta / K}, 0)$ of the separatrix, the inter-well chaotic motion pervades two wells and their vicinity.

Secondly, the classic SIM branch can better describe the high energy behaviours of bistable NES. The phase trajectory oscillates around the right branch of the SIM when the system shows SMR or stable response. By applying the classic complex variables $\phi_{1} e^{i \Omega \tau}=\dot{v}+i \Omega v, \quad \phi_{2} e^{i \Omega \tau}=\dot{w}+i \Omega w$ and a multiple-scales method, the traditional SIM can be extracted as in (19).

$$
\begin{aligned}
& Z_{1}=\lambda_{2}^{2} Z_{2}+(\delta-1)^{2} Z_{2}+\frac{3 K}{2}(\delta-1) Z_{2}^{2}+\frac{9 K^{2}}{16} Z_{2}^{3} \\
& Z_{1}=N_{1}^{2}, \quad Z_{2}=N_{2}^{2}
\end{aligned}
$$

This method can be found in various references. The unstable regime is divided by the singular value $Z_{2 i}$ :

$$
Z_{2 i}=\frac{4\left(2(1-\delta) \mp \sqrt{(1-\delta)^{2}-3 \lambda_{2}^{2}}\right)}{9 K}, i=1,2
$$

\subsection{Principle of SMR trigger mechanism}

The four most important items of NES amplitude information can be drawn from the classic SIM structure: (a) line A: attractor $-\delta / K$, (b) line B: chaos threshold $-2 \delta / K$, (c) line C: left stable region boundary $Z_{21}$, (d) line D: right 
stable region boundary $Z_{22}$. These four typical lines are marked in Fig. 9. Their relative positions will vary for different negative stiffnesses, which are mainly responsible for the characteristics of the regimes.

The four lines divide the $Z_{1}$ and $Z_{2}$ plane into two unstable regions. The interval of the first chaotic region is to the left of line B in Figure. 9(a) and is shaded. Once the phase trajectory goes beyond line B, the chaotic motion will cover this region, as in case 2 of Fig. 8(c). The second unstable region is the SIM unstable region that occupies an interval between lines $\mathrm{C}$ and $\mathrm{D}$, where the phase trajectory will deviate towards the left attractor or the SIM right branch.

The chaotic region will expand and move to the right as negative stiffness increases. The chaotic region will even overlap the SIM unstable region partially or entirely if the negative stiffness is intense enough, for example, Fig. 12(a). The size of the overlapping parts of the two areas determines the division of the response regimes that will be classified and discussed in the following section.

Generally, there are 3 fundamental criteria to describe the $Z_{1}, Z_{2}$ trajectory of the system:

(a) The trajectory starts from the attractor line A.

(b) Chaos occurs when the trajectory makes contact with chaos threshold line B.

(c) If the trajectory reaches line D, it jumps definitively to the right stable branch of the SIM.

Based on the above three criteria, all the response regimes that exist in bistable NES under harmonic excitation can be deduced.

\subsection{Classification of bistable NES}

The bistable NES preserves some original features of the cubic NES if a small value of negative stiffness is introduced. The distribution of efficiency under inputs of continually increasing energy is presented in Fig. 9, for a better comprehension of the distribution of the regimes. Its efficiency ratio is defined as follows:

$$
\begin{aligned}
& E_{L O}(\tau)=\int_{\tau_{0}}^{\tau} \epsilon \lambda_{1} \dot{x}^{2} d \tau, E_{N E S}(\tau)=\int_{\tau_{0}}^{\tau} \epsilon \lambda_{2}(\dot{x}-\dot{y})^{2} d \tau, \\
& r_{N E S}=\frac{E_{N E S}}{E_{L O}+E_{N E S}} \times 100 \%
\end{aligned}
$$

\subsubsection{Weak bistable NES}

Fig. 7 shows that the whole excitation range has five distinct phases. For each phase, the typical behaviour of the time domain and its phase trajectory are extracted in Fig. 8.

When the NES maintains an intra-well oscillation, e.g. case 1 in Fig. 8(c), this low energy level motion is trapped in one of the wells. Because the NES vibrates in the vicinity of equilibrium, the trajectory is quasi-asymmetric around 

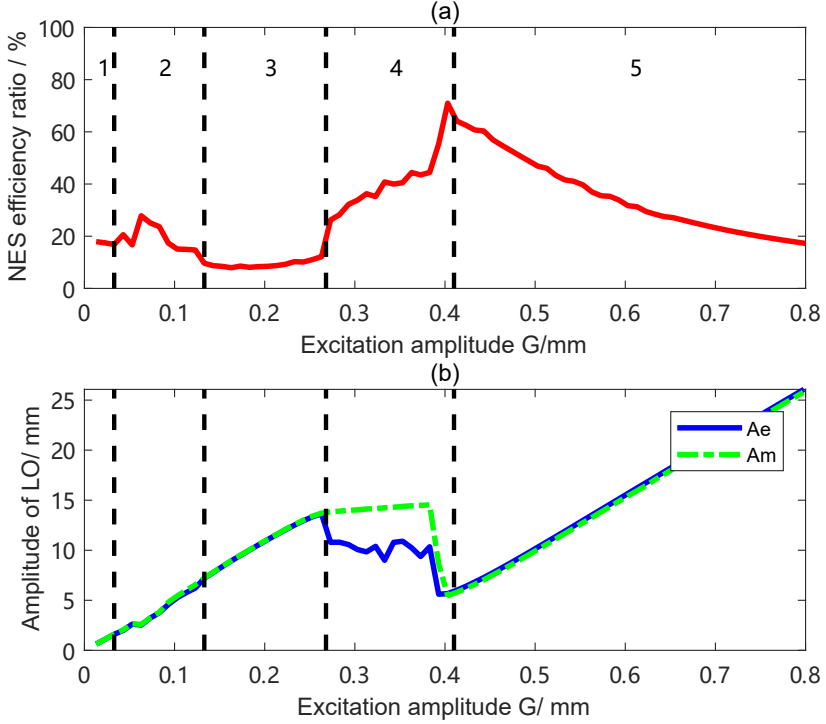

Fig. 7 Energy dissipation ratio of NES (a) and amplitude of LO (b) in weak bistable NES case. The blue line represents the average amplitude in a given time interval, the green dotted line is the maximum amplitude. The black dotted lines divide regimes into five stages
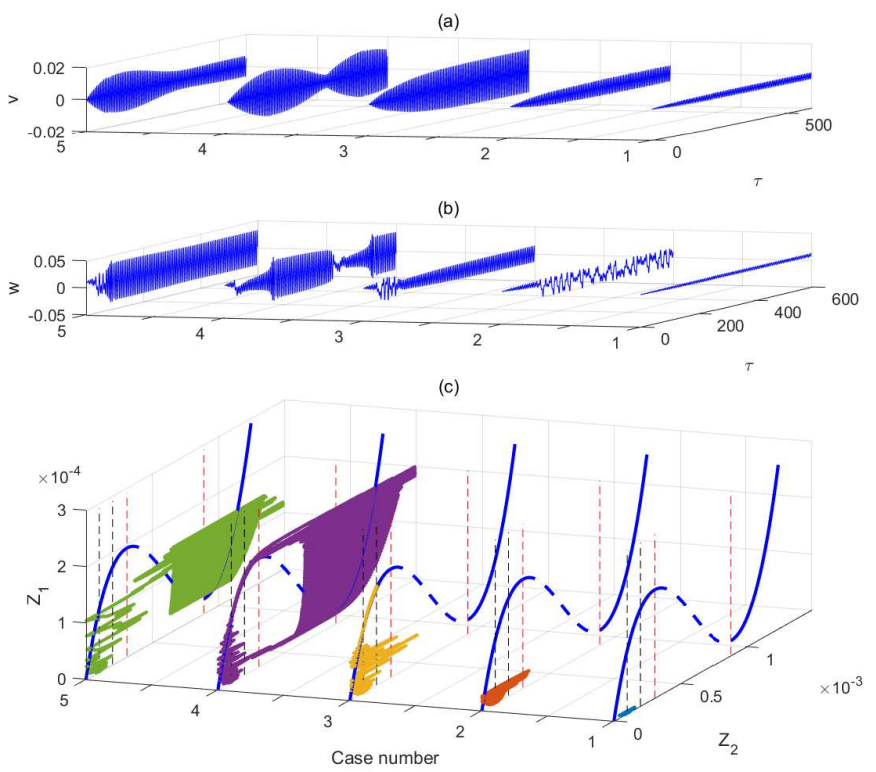

Fig. 8 Response regimes in weak bistable NES (a) v displacement (b) w displacement (c) phase trajectory of $Z_{2}$ and $Z_{1}$. The 5 typical responses are chosen at various excitations $\mathrm{G}$ $=0.04 \mathrm{~mm}, 0.1 \mathrm{~mm}, 0.25 \mathrm{~mm}, 0.35 \mathrm{~mm}, 0.42 \mathrm{~mm}$ 

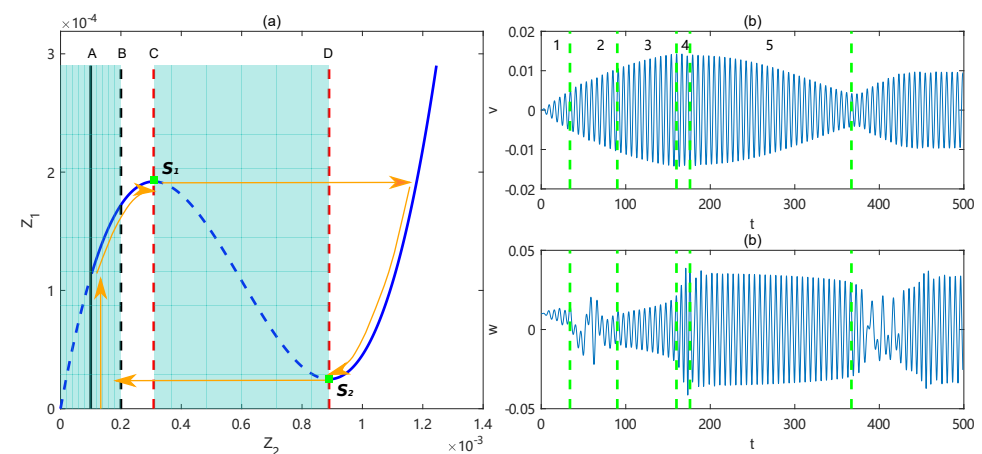

Fig. 9 Characteristic weak bistable SIM and SMR in the time domain. (a) The SIM structure with unstable region (shaded). Orange arrow line indicates various stages in one SMR cycle. $S_{1}$ and $S_{2}$ are the singular points whose locations are defined as $\left(Z_{11}, Z_{21}\right)$ and $\left(Z_{12}, Z_{22}\right)$. (b) displacement of $\mathrm{w}$, and (c) displacement of $\mathrm{v}$. Green lines divide the SMR into various stages corresponding the SIM explanation by orange arrow line.

attractor line A. The adapted complex variables method can describe its behaviours better by the local SIM according to the previous section.

In the case 2 of Fig. 8, increasing energy input causes the NES amplitude to exceed line B and trigger chaotic motion. However, the small value of $\delta$ leads to a significant gap between the chaos threshold line B and singular line D. The phase trajectory can neither activate SMR nor be attracted to the left SIM branch, but can only expand and take a position near line B, as in case 2 in Fig. 8(c). During the extension of the chaotic region in the $Z_{1}$, $Z_{2}$ planes, there are two expansion directions, $Z_{1}$ direction and $Z_{2}$ direction, respectively. If the distance between the phase trajectory and the left stable SIM branch is much smaller than the distance between the phase trajectory and the singular line $\mathrm{D}$, there is a high probability of the phase trajectory of the system being attracted to the stable solution of the left stable SIM branch, instead of snap-through motion (SMR).

In the third stage of Fig. 7, after the generation and transient expansion of chaos, the time domain displacement of $\mathrm{w}$ is symmetrical to the zero position. The phase trajectory is re-attracted to the left stable SIM branch as in case 3 in Fig. 8(c) and climbs up along the left SIM branch. This attraction motion that results from the phase trajectory increasing in the $Z_{1}$ direction affects the left stable SIM branch more quickly than the expansion of the phase trajectory in $Z_{2}$ direction in the initial low energy input stage. The amplitude of LO increases linearly with increasing $G$ in the first 3 stages in Fig. 7(b) and the corresponding efficiency maintains a low level, which implies that the TET is not activated.

In the fourth stage of Fig. 7, a complete SMR emerges. The time domain divided by green dashed lines in Fig. 9(b) shows five different parts of a complete SMR: (1) intra-well oscillation, (2) Chaos expansion, (3) Re-attraction to SIM, (4) Jumping motion, (5) targeted energy transfer. Compared with the 
SMR in the pure cubic case, the SMR starts from the intra-well oscillation, so the orange arrow line represents a trajectory climbing along line A in Fig. 9(a). The initial motion is constrained in the well and increases until the trajectory is retracted to the left stable SIM branch, on which the orange lines converge. As the trajectory crosses the singular line C, it jumps to the right stable branch of the SIM, and moves down to the other singular point $S_{2} .1: 1$ resonance in this period produces an intense targeted energy transfer and leads to effective dissipation by the NES. Once the NES has dissipated most of the energy of the LO, the phase trajectory of the system jumps back to the chaotic region in the vicinity of attractor line A and waits for the charge of energy under harmonic excitation. Due to the presence of an efficient dissipative phase, the SMR has relatively high efficiency.

In the fifth stage of Fig. 7, the phase trajectory in the chaotic region has a larger amplitude like case 5 of Fig. 8(c), resulting in the trajectory easily crossing the SIM unstable region. Chaos triggers the SMR. In contrast to the fourth stage of efficieny distribution, the enormous energy input case lacks the stage where the trajectory climbs along the left stable SIM branch, which is referred to as the 'charge energy stage'. The trajectory jumps directly to the right SIM branch before it reaches maximum value $Z_{11}$ or $S_{1}$ point. So the maximum amplitude of LO at the jump moment is lower than that of the SMR stage. This implies that the primary system can be protected better if there is high energy input in a weak bistable NES case. Since the phase trajectory finally becomes located on the right branch of the SIM without jumping back, this indicates saturation of the capability of the NES to absorb energy. The final position of the phase trajectory will be located in a higher position of the right SIM branch with an excitation of increasing amplitude. The optimal point ideally occurs at the singular point $S_{2}$. The optimal point is interpreted by the efficiency distribution figure in the transition between SMR and stable response period.

\subsubsection{Modest bistable NES}

The re-attraction to the SIM mechanism in a weak bistable system becomes delicate when the $\delta$ parameter takes a larger value. The mechanism of this attraction back to the left SIM branch is mainly due to the proximity of the chaotic trigger line B to the left SIM branch. The phase trajectory has a strong possibility of continuing to expand along with line A and being attracted by the left SIM branch, rather than crossing the unstable region and triggering SMR. If the negative stiffness is intense enough, the chaos trigger line B will be located in the SIM unstable region. Therefore, the critical condition for the disappearance of re-attraction can be expressed as follows:

$$
\begin{aligned}
& Z_{21}=\frac{4\left(2(1-\delta)-\sqrt{(1-\delta)^{2}-3 \lambda_{2}^{2}}\right)}{9 K}=\frac{-2 \delta}{K} \\
& \delta_{w m}=-\frac{8}{7} \pm \frac{2}{7} \sqrt{-7 \lambda_{2}^{2}+9}
\end{aligned}
$$

The damping of the NES system determines the critical value of negative stiffness. If the negative critical value exceeds the critical value -0.295 (when 

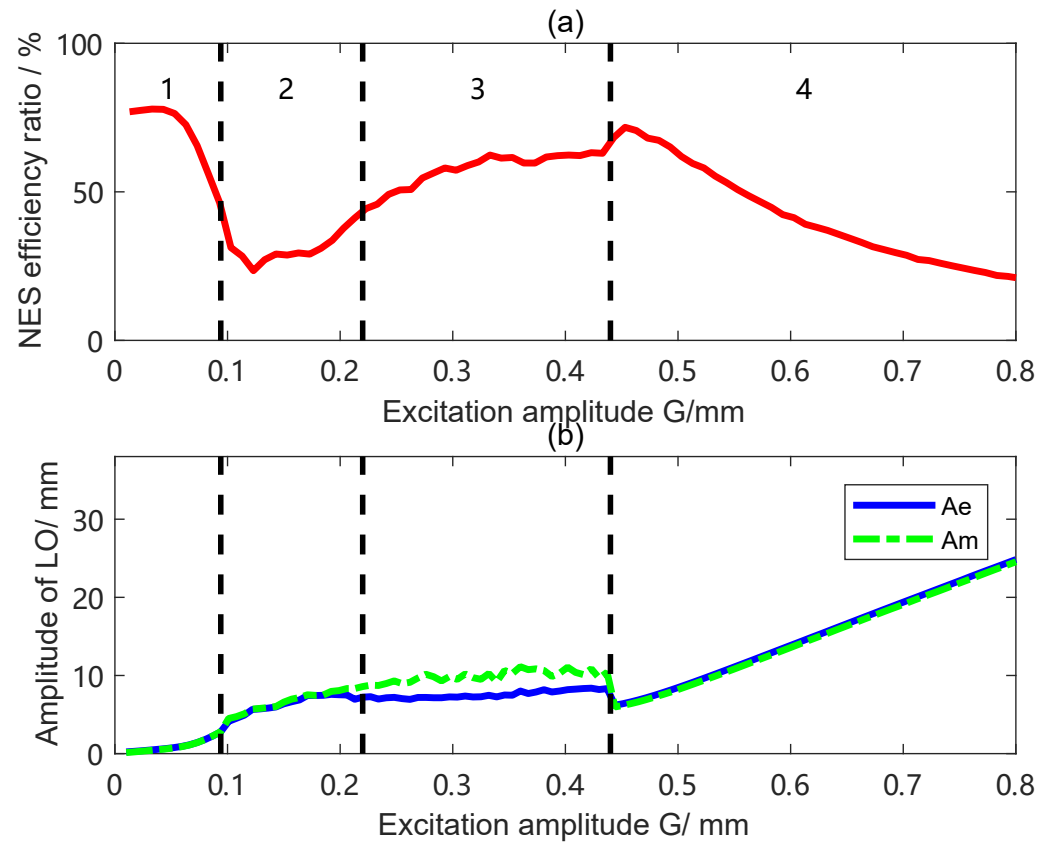

Fig. 10 Energy dissipation ratio of NES (a) and amplitude of LO (b) in modest bistable NES case. The blue line represents the average amplitude in a given time interval, the green dotted line is the maximum amplitude

$\left.\lambda_{2}=0.167\right)$, the re-attraction to the left SIM branch mechanism is hardly observable. To better prove this point, the efficiency distribution for a larger negative stiffness case is presented in Fig. 10. This larger negative stiffness case $\left(k_{3}=-60, \delta=-0.52\right)$ is called a modest bistable NES to distinguish it from the case in the previous section. The efficiency distribution can be divided into 4 stages in Fig. 10 and its characteristic behaviours under inputs of increasing amplitude excitation are presented in Fig. 11.

During the intra-well oscillation stage, the NES possesses a high absorbing efficiency because of the nonlinear resonance. However, as the excitation amplitude increases, its high efficiency is lost and declines drastically.

In the second stage in Fig. 10, chaos emerges. When $\delta$ increases, the span and depth of potential well become larger, so a larger amplitude excitation is necessary to trigger the chaotic motion. The critical value is $G=0.09 \mathrm{~mm}$, which divide the efficiency distribution figure into the chaotic and intra-well region in Fig. 10, while the chaos threshold excitation is $\mathrm{G}=0.03 \mathrm{~mm}$ in a weak bistable case. A coexistence of subharmonic oscillations and chaotic motions can be realised in this stage.

The SMR occupies the third stage in Fig. 10. For an SMR cycle of the modest bistable case, the attraction to the SIM part 3 in Fig. 9(b), 9(c) has been completely compressed and replaced by the SMR and chaos expansion. Only 
(a)

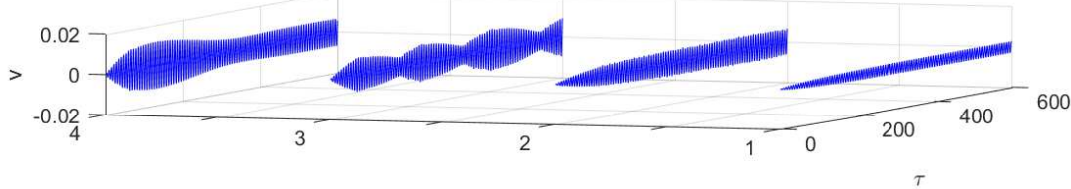

(b)

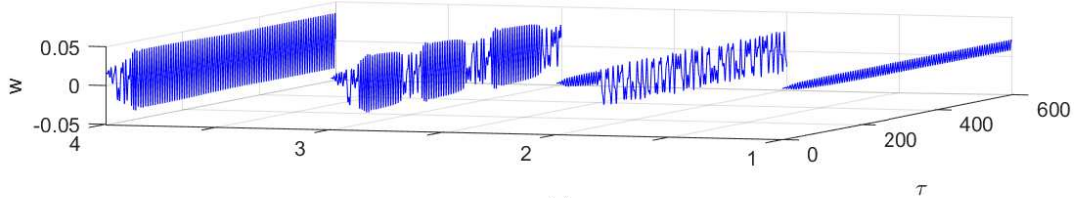

(c)

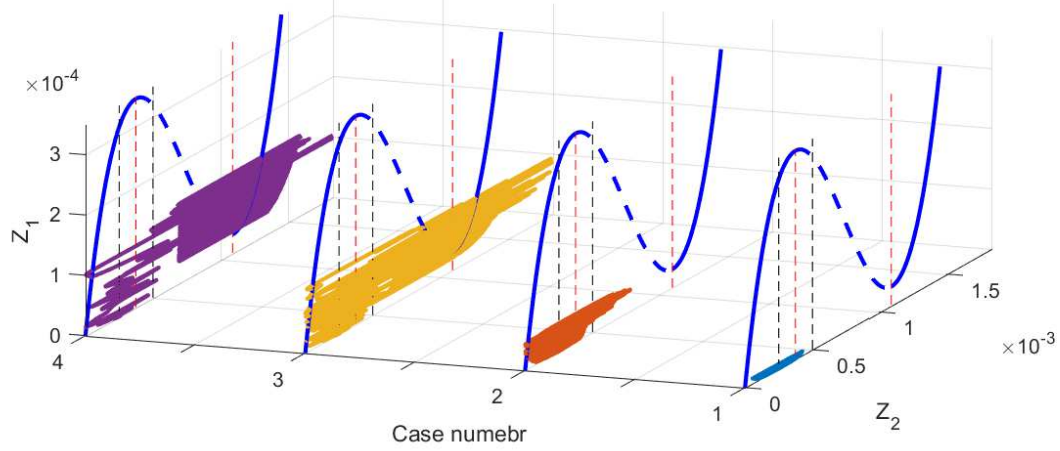

Fig. 11 Response regimes in modest bistable NES (a) v dispalcement (b) w displacment (c) phase trajectory of $Z_{2}$ and $Z_{1}$. The 4 typical responses are chosen at various excitations $\mathrm{G}=0.08 \mathrm{~mm}, 0.15 \mathrm{~mm}, 0.34 \mathrm{~mm}, 0.45 \mathrm{~mm}$.
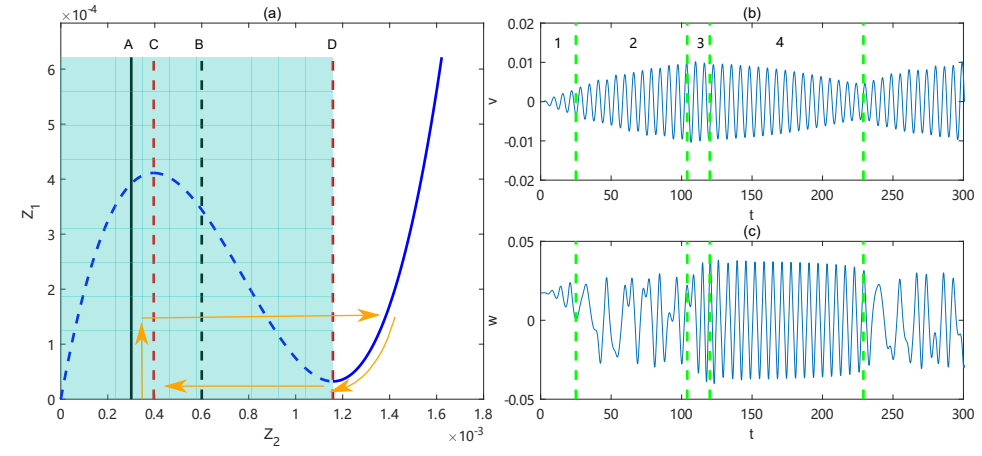

Fig. 12 Characteristic modest bistable SIM and SMR in the time domain. (a) The SIM structure with the unstable region (shaded). The orange arrow line indicates various stages in one SMR cycle. (b) displacement of $\mathrm{w}$, (c) displacement of $\mathrm{v}$. The green lines divide the SMR into various stages corresponding to the SIM explanation by the orange arrow line 
four stages have been retained in Fig. 12(b), 12(c) : (1) intra-well oscillation, (2) Chaos expansion, (3) Jumping motion, (4) targeted energy transfer.

In Fig. 12(a), a greater value of negative stiffness leads to the fact that the chaos trigger line B is located in the SIM unstable region and is close to the singular line D. It can be deduced that SMR is more early to produce in modest bistable case. As already observed in Fig. 10(b), the SMR region starts at $\mathrm{G}=0.22 \mathrm{~mm}$, which is lower than the $\mathrm{SMR}$ trigger excitation $\mathrm{G}=0.26 \mathrm{~mm}$ in weak bistable NES.

Because the extreme right point in phase trajectory of case 2 is close to singular line $D$ in Fig. 11(c), the SMR is trigged by crossing chaotic region and unstable SIM region in $Z_{1}, Z_{2}$ plane instead of reaching at the singular point $S_{1}$ and then jumping. It means that the system does not require fully charging the energy to activate SMR. A lower trigger excitation amplitude results in a lower initial $Z_{1}$ amplitude, from which the trajectory moves down along the right stable SIM branch. This shorter path helps NES dissipate energy around the optimal point within a shorter time and higher efficiency. As the case 3 in Fig. 11(c), the system performs 3 SMR cycles within $600 \tau$. However, the weak bistable case performs only one complete SMR at the same time. Chaos provides a much faster way to charge and trigger SMR to accelerate every SMR circle. More SMRs in a fixed time interval are observed in Fig. 11(c). That is why the SMR stage, the modest bistable NES, has higher efficiency than that of weak bistable NES. A more efficient way to dissipate energy is generated.

In the fourth stage, the NES system possesses a stable regime again. The optimal point is generated in this stage. The absorption efficiency of the NES system decreases with increasing external excitation in Fig. 10.

\subsubsection{Strong bistable NES}

The chaos threshold B line will approach the SMR boundary line D more closely for higher negative stiffness. When chaos first appears, the amplitude of the NES will exceed line B. If the distance between lines B and D is small enough in Fig. 15, the expansion of the chaos regime will disappear in effeciency distribution Fig. 13. The trajectory will cross the singular line D and directly start to jump and perform an SMR. Case 2 in Fig. 14 shows that the snapthrough phenomenon occurs at the instant when chaos appears. The strong bistable NES is defined as the NES for which chaos expansion vanishes. This critical negative stiffness value is equal to -1.05 , which is obtained by numerical simulation. The energy dissipation ratio can be classified into 3 stages: (1) intra-well oscillation, (2) SMR, (3) stable stage.

In the intra-well stage, the absence of nonlinear beating leads the NES system to possess low efficiency. The energy is mainly absorbed by the LO, the amplitude of which mainly increases linearly in Fig. 13(b).

In the SMR stage, an SMR cycle can be divided into 3 parts: (1) intra-well oscillation, (2) snap-through, and (3) targeted energy transfer as in Fig. 15(b), 
(a)

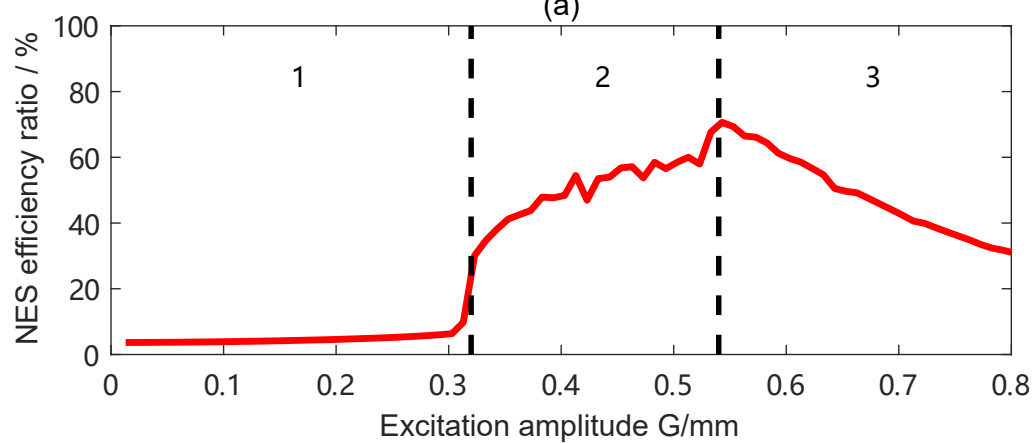

(b)

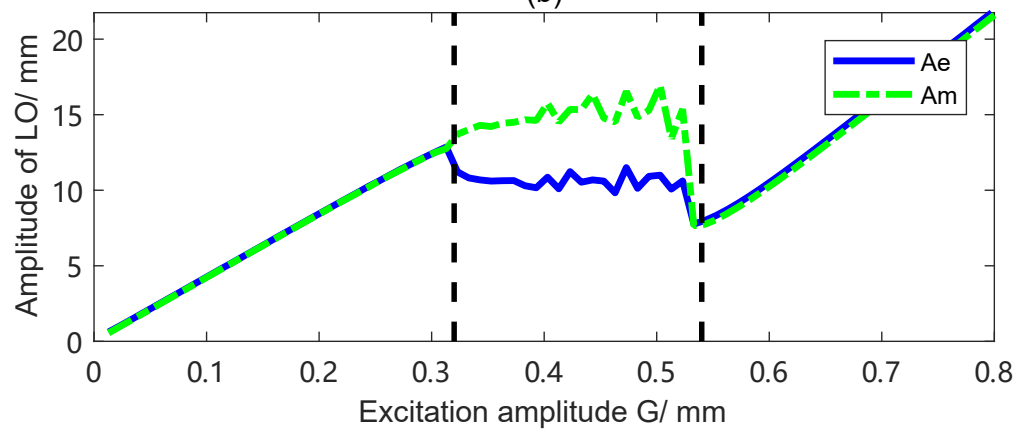

Fig. 13 Energy dissipation ratio of NES (a) and amplitude of LO (b) in strong bistable NES case. The blue line represents the average amplitude in a given time interval, the green dotted line is the maximum amplitude

$15(\mathrm{c})$. Once the phase trajectory crosses the chaos trigger line $\mathrm{B}$, the right stable branch of the SIM attracts the phase trajectory. This leads to the simultaneous appearance of the SMR and chaos. The chaotic motion is obvious when the phase trajectory jumps back to the chaotic region. An enormous value of $\delta$ requires more energy to escape the well. With the same damping condition, a longer 'charge time' (intra-well oscillation) is required before snap-through is attained, as in case 2 of Fig. 14(b). The motion of this stage is either in a potential well or in the right stable SIM branch and the chaotic motion becomes weak and transient. Compared with SMR stage in weak bistable NES case, the duration of energy pumping in Fig. 14(b) is longer and it has a less absorbing cycle within the same time interval.

In a stable response stage, the large negative stiffness value increases the force threshold for escaping from a potential well. The charging process will be a little shorter under higher energy input. The effect of the attractor restricts the motion before the jump out of one of the potential wells takes place. 
(a)

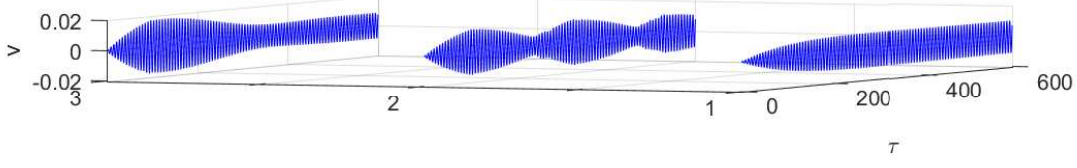

(b)

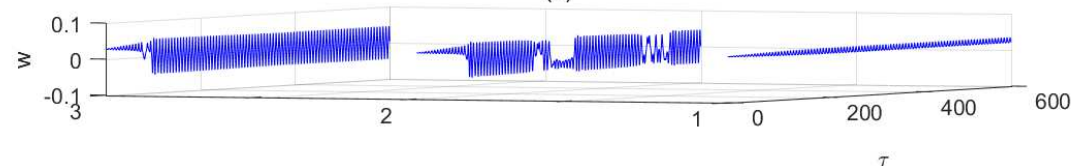

(c)

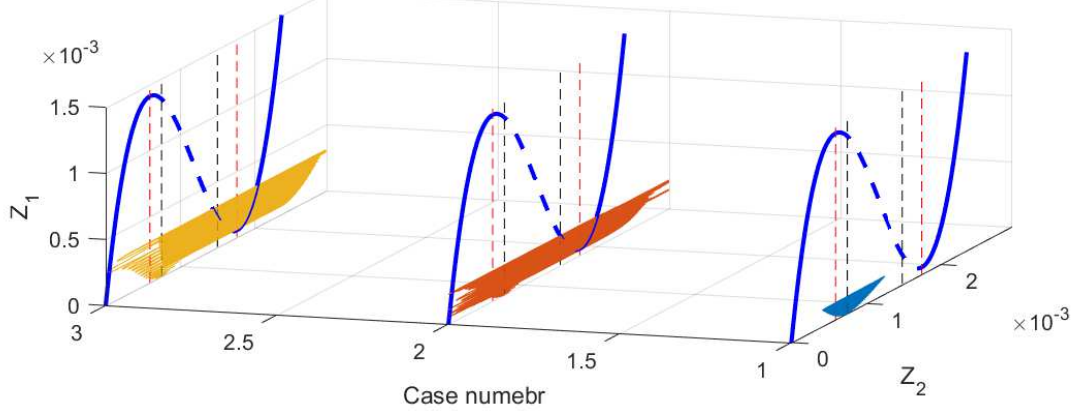

Fig. 14 Response regimes in strong bistable NES (a) v displacement (b) w displacement (c) phase trajectory of $Z_{2}$ and $Z_{1}$. The 3 typical responses are chosen at various excitations $\mathrm{G}=0.25 \mathrm{~mm}, 0.45 \mathrm{~mm}, 0.55 \mathrm{~mm}$
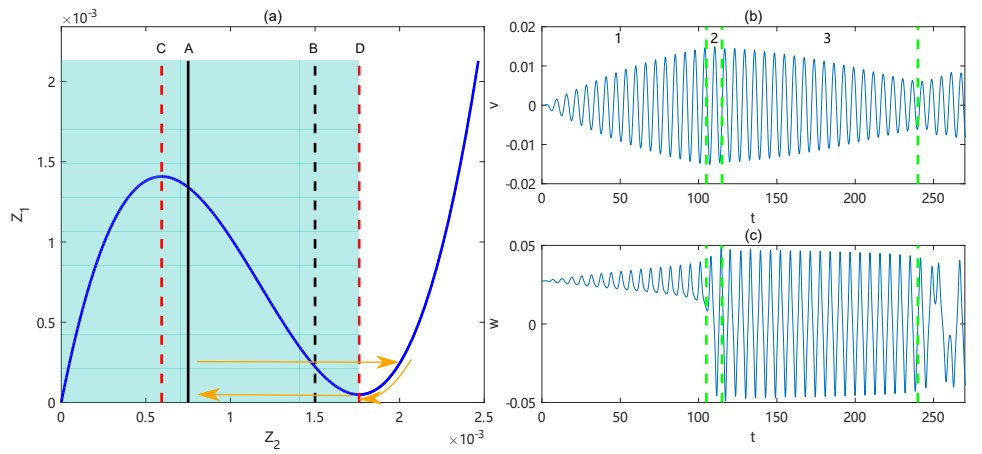

Fig. 15 Characteristic strong bistable SIM and SMR in the time domain. (a) The SIM structure with the unstable region (shaded). The orange arrow line indicates various stages in one SMR cycle. (b) displacement of w, (c) displacement of $\mathrm{v}$. The green lines divide the SMR into various stages corresponding to the SIM explanation indicated by the orange arrow line 


\subsubsection{Abnormal bistable NES}

If the negative stiffness is extremely large, another critical condition can be achieved, where the chaos threshold line $\mathrm{B}$ coincides with the line $\mathrm{D}$, and the following equation can be derived:

$$
\begin{aligned}
& \frac{4}{9} \frac{2-2 \delta+\sqrt{(1-\delta)^{2}-3 \lambda_{2}^{2}}}{K}=-\frac{2 \delta}{K} \\
& \delta_{w m 2}=-\frac{8}{7} \pm \frac{2}{7} \sqrt{-7 \lambda_{2}^{2}+9}
\end{aligned}
$$

Solving the above equation gives a critical negative stiffness of -2 . In this case, the trajectory exceeds the chaos threshold and becomes a stable inter-well oscillation. Because the $Z_{1}$ amplitude is then close to the singular value $Z_{12}$, the local minimum in the right SIM branch, the SMR vanishes. Only two regimes persist in the efficiency distribution and amplitude of LO in Fig. 18 : (1) Intrawell oscillation, and (2) Stable response. In contrast to the previous model, the SMR stage is compressed and vanishes, leading to a so-called abnormal bistable NES.

The trigger chaos line $\mathrm{B}$ has exceeded enormously on the right side of singular line D, so the optimal point (maximum efficiency) is not lying on the singular point $S_{2}$. The maximum efficiency that an abnormal NES can achieve is much lower than in previous cases.

\section{Experimental validation}

The goals of the experimental tests were to verify the feasibility of the intrawell adapted complex variables method in the frequency domain and to observe the characteristic response regimes under increasing excitation amplitude inputs. Various negative stiffnesses were constructed by adjusting the pre-compression length of the linear spring in the bistable NES. A diagram of the bistable NES and the actual experimental device are presented in Fig. 19 and 20 .

The bistable stiffness is constructed by combining 2 linear springs and 2 conical springs that mainly provide the nonlinear stiffness. The conical spring presents two phases: (a) linear phase, (b) nonlinear phase during the compression [14]. When the coils of a conical spring come into contact with each other due to compression, a transition moment occurs that divides the linear and nonlinear phases. So the two conical springs are pre-compressed at the transition point to eliminate the linear phase, as in (a) of Fig. 19. The two linear springs, whose role is to counterbalance the linear stiffness in the nonlinear phase, are installed perpendicular to the conical springs like (b) in Fig. 19. The force-displacement relation of combining system can be expressed as follows:

$$
\begin{aligned}
& F=k_{2} u+k_{3} u^{3} \\
& k_{2}=\left(a_{1}+k_{0}-2 k_{l} \frac{l_{p}}{l_{0 l}+2 l_{c}-l_{p}}\right), k_{3}=\left(a_{3}+k_{l} \frac{l_{0 l}+2 l_{c}}{\left(l_{0 l}+2 l_{c}-l_{p}\right)^{3}}\right)
\end{aligned}
$$


(a)

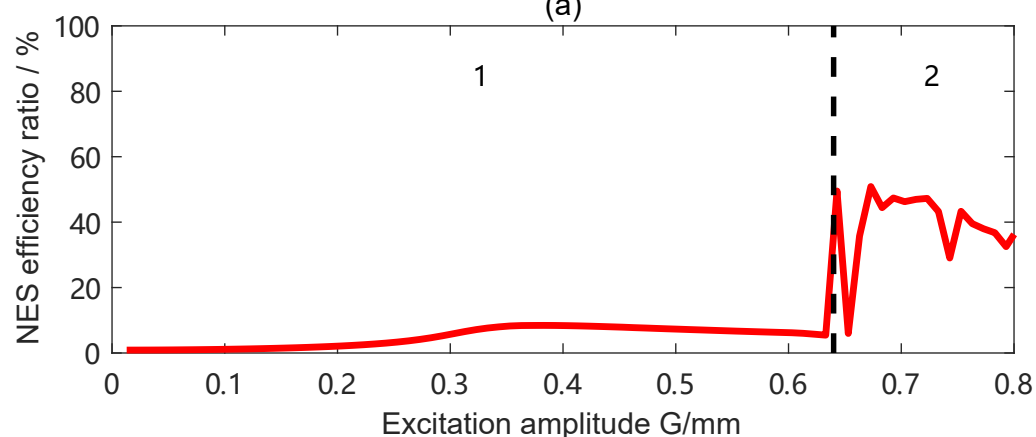

(b)

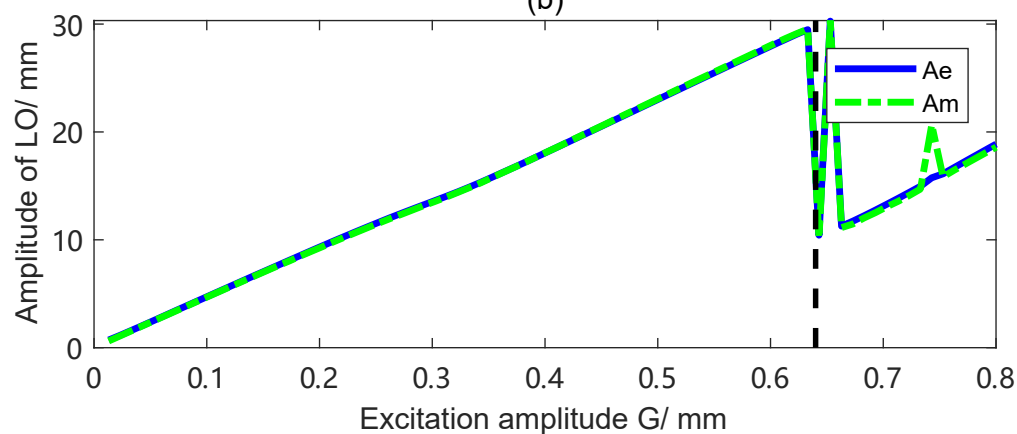

Fig. 16 Energy dissipation ratio of NES (a) and amplitude of LO (b) in abnormal bistable NES case

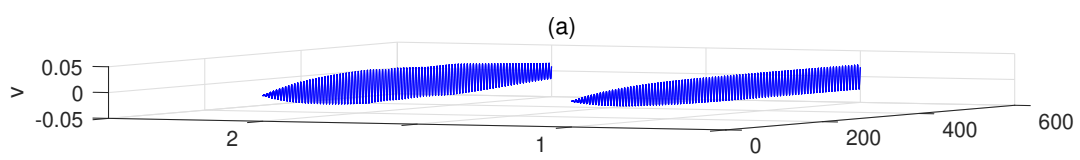

(b)
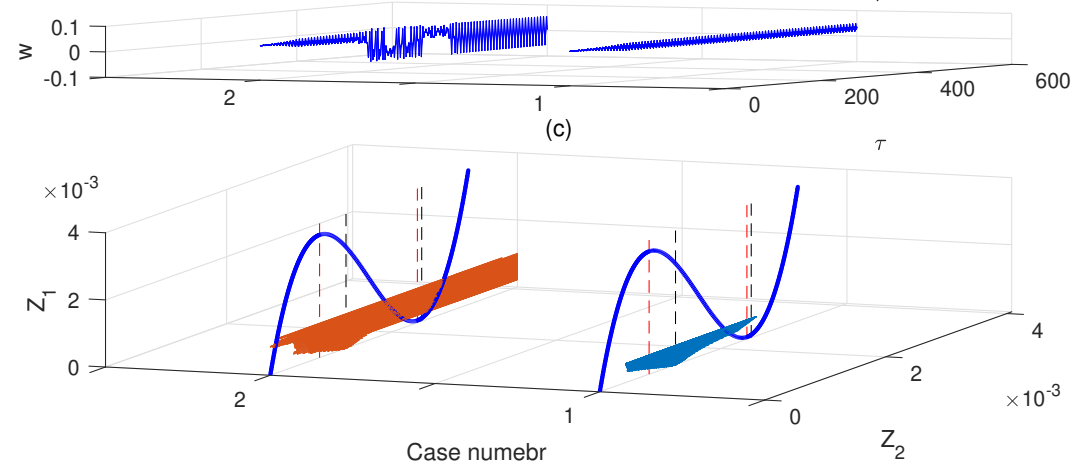

Fig. 17 Response regimes in strong bistable NES (a) v displacement, (b) w displacement, (c) phase trajectory of $Z_{2}$ and $Z_{1}$. The 2 typical responses are chosen at various excitations $\mathrm{G}=0.5 \mathrm{~mm}, 0.7 \mathrm{~mm}$. 

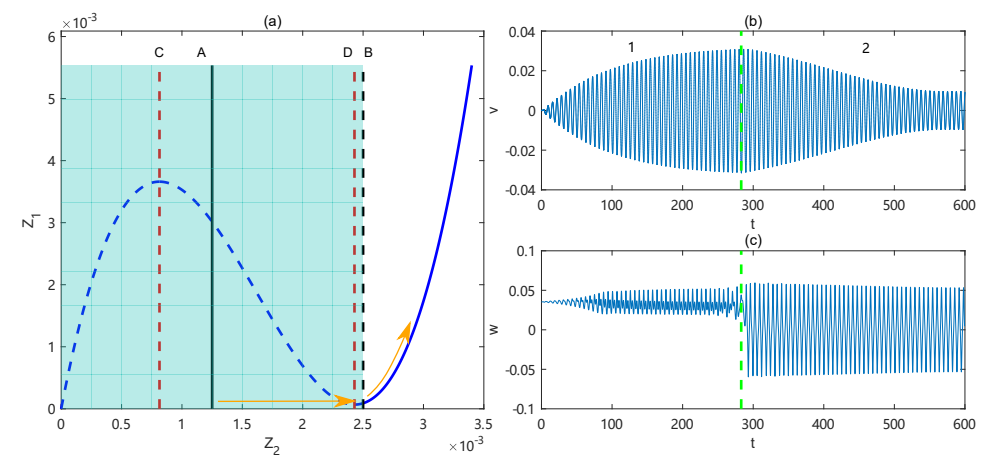

Fig. 18 Characteristic abnormal bistable SIM and SMR in the time domain. (a) The SIM structure with unstable region (shaded). The orange arrow line indicates various stages in one SMR cycle. (b) displacement of w; (c) displacement of $\mathrm{v}$. The green lines divide the SMR into various stages corresponding the SIM explanation by the orange arrow line.

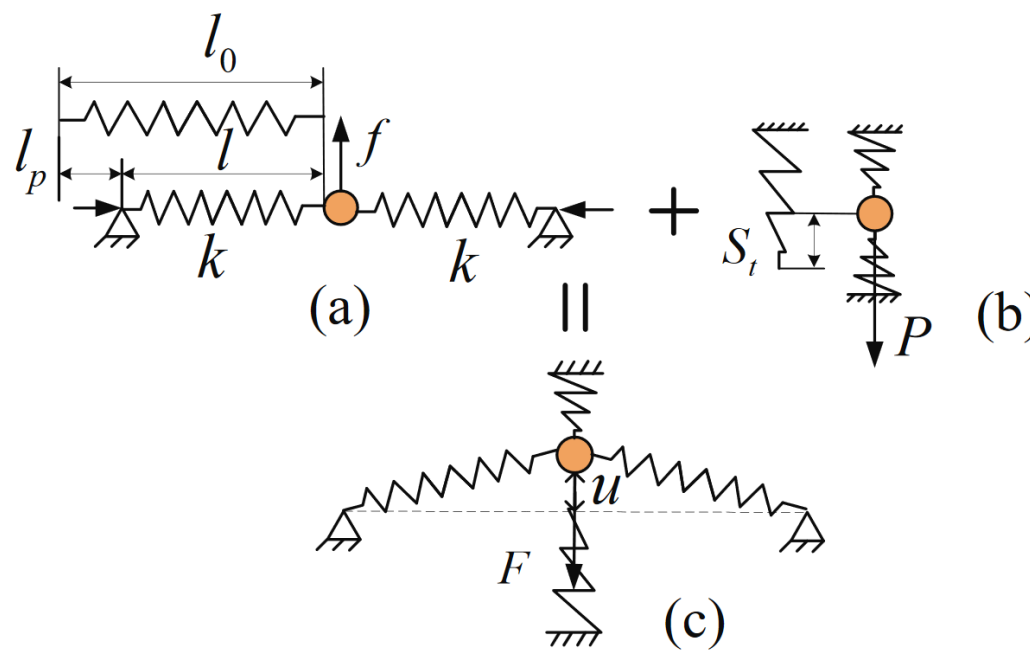

Fig. 19 Detailed diagram of the bistable NES system: (a) negative stiffness mechanism, (b) conical spring system, (c) combining system

where $k_{0}$ represents the linear phase stiffness and $a_{1}, a_{3}$ are the linear stiffness and cubic stiffness in the nonlinear phase of a conical spring. $l_{0 l}$ and $l_{c}$ are the lengths of the linear spring and connector, respectively. $k_{l}$ is the stiffness of the linear spring. The pre-compression length $l_{p}$ determines the value of both the negative stiffness $k_{3}$ and the nonlinear stiffness $k_{2}$. The detailed parameters and 3 initial pre-compression lengths for 3 different bistable NES cases are presented in Table. 1.

The corresponding static force-displacement figures for each case are presented in Fig. 21. In each case, the theoretical result provides sufficient accuracy to describe the experimental result and two equilibria $(F=0)$, one on 


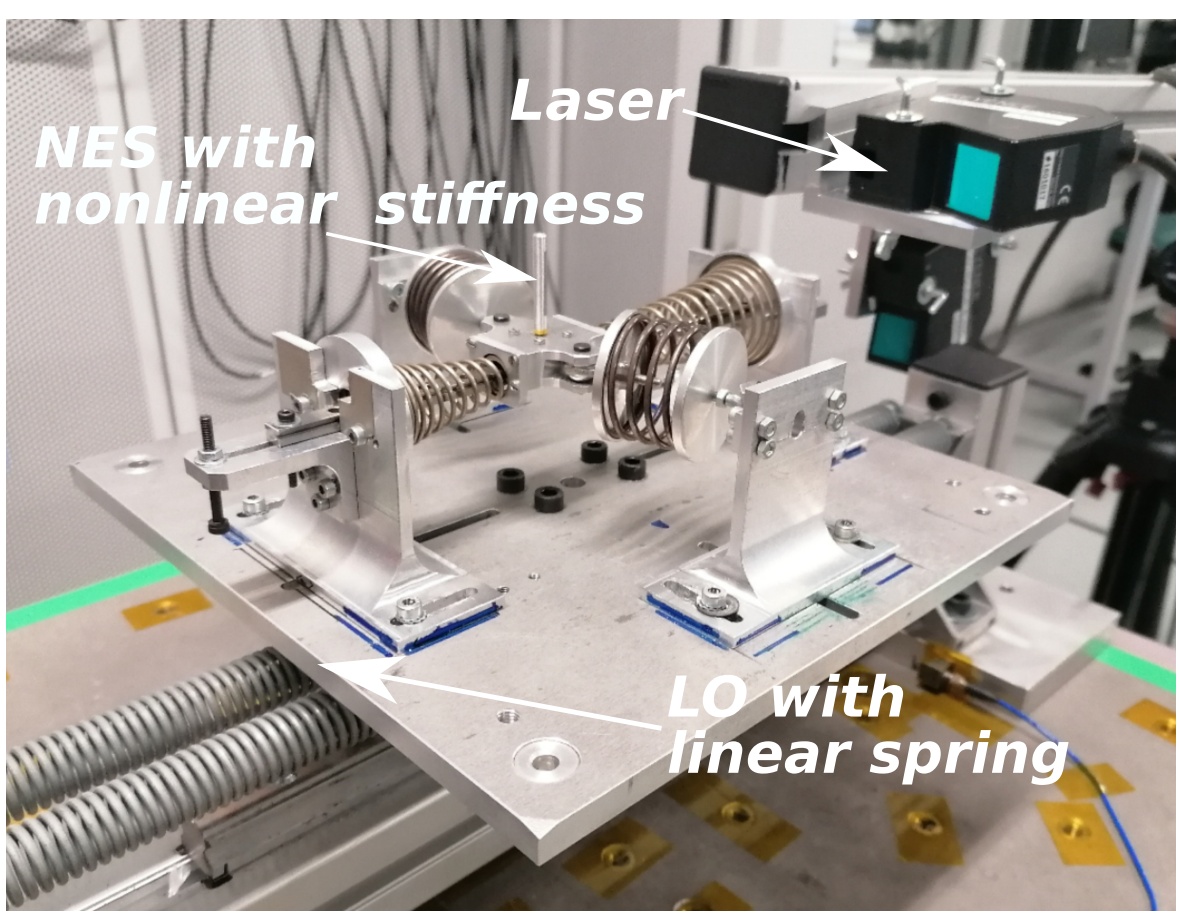

Fig. 20 Global view of the experimental setup. The four spring system constructs the nonlinear stiffness. LO is connected to a shaker by the linear spring and vibrates in a track

Table 1 Experimental parameters of NES system

\begin{tabular}{lllll}
\hline$k_{0}$ & $a_{1}$ & $a_{3}$ & $k_{l}$ & $l_{0 l}$ \\
$187 \mathrm{~N} / \mathrm{m}$ & $280 \mathrm{~N} / \mathrm{m}$ & $3.6 \mathrm{e} 5 \mathrm{~N} / \mathrm{m}^{3}$ & $1060 \mathrm{~N} / \mathrm{m}$ & $50 \mathrm{~mm}$ \\
\hline & $l_{c}$ & $l_{p 1}$ & $l_{p 2}$ & $l_{p 3}$ \\
& $14.5 \mathrm{~mm}$ & $16 \mathrm{~mm}$ & $17.5 \mathrm{~mm}$ & $21 \mathrm{~mm}$ \\
\hline
\end{tabular}

either side of the displacement, which characterize a bistable NES. The distance between the equilibrium points becomes greater when the $l_{p}$ increases, resulting in increased span and depth of the potential well. So it can be concluded that the control strategy of changing the length of pre-compression to produce desirable stiffness characteristics is feasible. However, increasing $l_{p}$ will not only increase the value of $\delta$ but also cause an augmentation of $K$, which is different from the idea of purely introducing $\delta$ and keeping $K$ constant used in the previous bistable NES classification.

The testing system consisted of a NES embedded with a LO. A $10 \mathrm{kN}$ electrodynamic shaker provided the excitation at a variable frequency. The absolute displacements of NES and LO were measured by two laser systems installed vertically. The bandpass filter filtered the high-frequency noise, thus correcting the raw signal and the biases. The amplitude of excitation was 0.08 


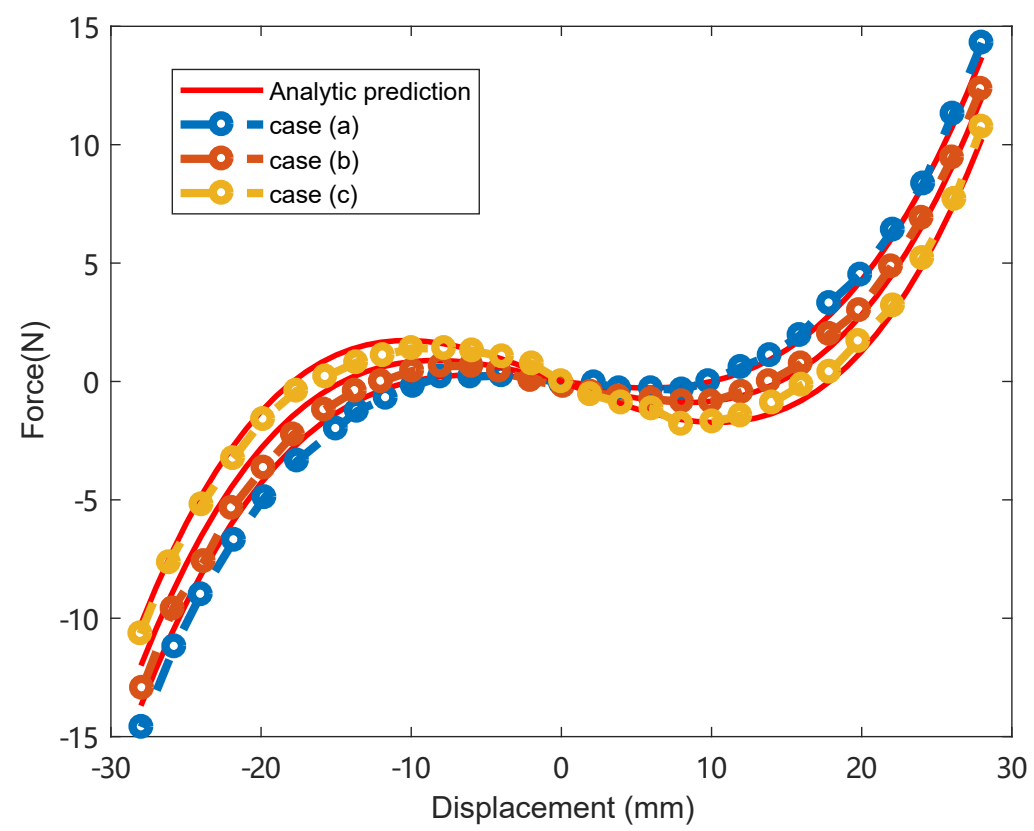

Fig. 21 Force displacement of different experiment tests with parameters in Table. 3

Table 2 Experimental parameters

\begin{tabular}{llllll}
\hline Physical parameters & $m_{1}$ & $m_{2}$ & $c_{1}$ & $c_{2}$ & $k_{1}$ \\
& 5.5 & 0.05 & 5 & 0.5 & $1.148 \mathrm{e} 4$ \\
\hline Reduced parameters & $\epsilon$ & $\lambda_{1}$ & $\lambda_{2}$ & $f_{0}$ & \\
& $0.91 \%$ & 2.19 & 0.22 & 7.27 & \\
\hline
\end{tabular}

$\mathrm{mm}$, which was the minimum value that the shaker could apply. Its frequency was varied from $7 \mathrm{~Hz}$ to $7.6 \mathrm{~Hz}$ at a sweep velocity of $0.01 \mathrm{~Hz} / \mathrm{s}$.

The mass of the NES was small, so the inertia of the springs was not negligible. The effective mass of a conical spring and a linear spring can be found in [14]. The viscous damping coefficient was estimated by modal analysis, where the nonlinear stiffness was replaced by linear stiffness. The physical parameters are summarized in Table 2 . The different negative stiffnesses caused by various pre-compression lengths are presented in Table 3

Fig. 22 shows the experimentally obtained frequency response function for the small amplitude excitation $\mathrm{G}=0.08 \mathrm{~mm}$, where 3 cases perform intra-well oscillation. The analytical result was obtained by substituting the reduced parameters in Tab. 2 and 3 into (6) and resolving the amplitude of LO $\left|\phi_{10}\right|$.

When a natural frequency excitation is applied in LO, the resonance phenomenon is activated. The LO possesses the largest amplitude, of $7.23 \mathrm{~Hz}$, close to the predicted value of $7.26 \mathrm{~Hz}$. In general, the analytical method de- 
Table 3 Experimental stiffness coefficients

\begin{tabular}{llll}
\hline & case (a) & case (b) & case (c) \\
\hline$k_{3}$ & -71.4 & -136.3 & -300.1 \\
$k_{2}$ & $6.95 \mathrm{e} 5$ & $7.2 \mathrm{e} 5$ & $7.89 \mathrm{e} 5$ \\
\hline$\delta$ & -0.68 & -1.31 & -2.89 \\
$K$ & $6.59 \mathrm{e} 3 \mathrm{e} 5$ & $6.90 \mathrm{e} 3$ & $7.58 \mathrm{e} 3$ \\
\hline
\end{tabular}
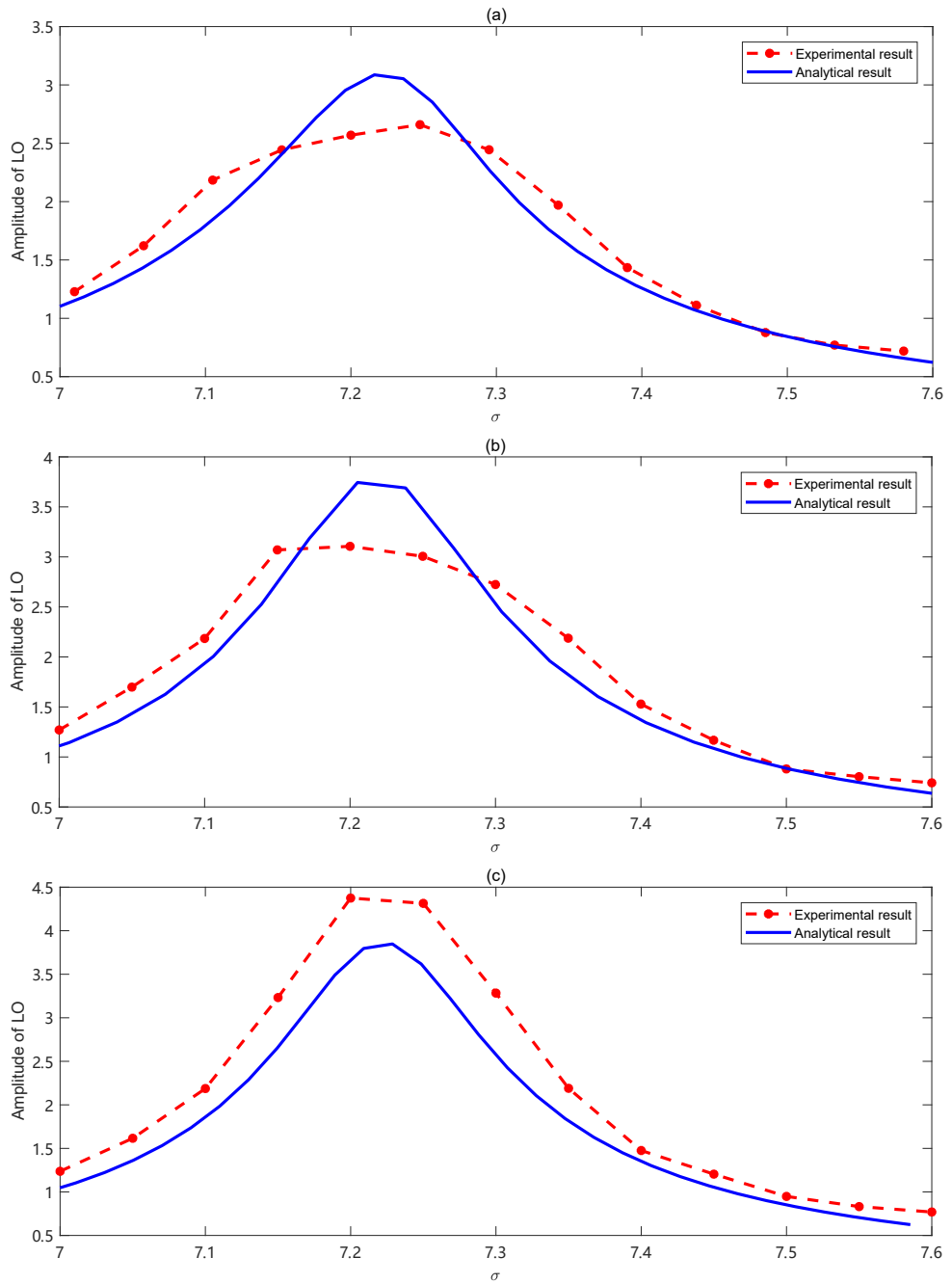

Fig. 22 Experimental and analytical frequency response curve of LO for different precompression length cases. The parameters of the 3 cases are presented in Table. 3 
scribed the intra-well oscillation correctly under various $\delta$ cases as shown in Fig. 22. The analytical amplitude, which is compared with the experimental result, had the same error distribution under different negative stiffnesses. In the vicinity of the natural frequency, the analytical result was usually greater than the experimental result. Beyond the natural frequency, the analytical result had a lower amplitude. This error distribution is the same as in the numerical test of Fig. 4, where the analytical method possesses a larger result near $\sigma=0$. In the most intensive $\delta$ case in Fig. 22(c), the calculation method gave a lower analytical result. This confirms the previous conclusion that the adapted complex variables method leads to minor error in the strong bistable case under small excitation. This is due to the fact that the negative stiffness value is too large to cause deformation of the real phase trajectory near the equilibrium point (which does not conform to the assumption of a circle).

\section{Response regimes in frequency domain}

The previous section described the response regimes in 1:1 transient resonance at various energy input levels. However, the advantage of a NES in absorbing energy is more apparent in the frequency domain. In this section, the response of the bistable NES is investigated experimentally over a broader range of frequencies.

The three different compression cases, having parameters that were identical to those of the previous intra-well experimental validation, were tested under a frequency sweeping excitation from $7 \mathrm{~Hz}$ to $7.6 \mathrm{~Hz}$. The same frequency sweeping process with different excitation amplitudes was repeated to record the responses of the LO and NES.

\subsection{Case (a) experiment}

11 sets of excitation amplitudes, from small to large values: $0.08 \mathrm{~mm}, 0.10$ $\mathrm{mm}, 0.12 \mathrm{~mm}, 0.15 \mathrm{~mm}, 0.18 \mathrm{~mm}, 0.21 \mathrm{~mm}, 0.25 \mathrm{~mm}, 0.28 \mathrm{~mm}, 0.32 \mathrm{~mm}$, $0.36 \mathrm{~mm}$, and $0.4 \mathrm{~mm}$, were tested for case (a). To help distinguish them, the adjacent time-displacement curves are marked with different colours.

In Fig. 23, the black diamond points distinguish the SMR region and resonance peak (potential risk case), where the amplitude of the LO is enormous, and the efficiency of absorbing energy fails for the NES.

Before the amplitude excitation increased to $0.21 \mathrm{~mm}$, the stable response was the primary behaviour. In the vicinity of the natural frequency, $7.26 \mathrm{~Hz}, 1: 3$ subharmonic oscillation occurred first for low energy input. Before the external excitation reached a threshold, the region of 1:3 subharmonic resonance broke and expanded to higher and lower frequency sides with increasing external force. In the neighbourhood of the natural frequency, the response reverted to a 1:1 resonance. The stability of subharmonic oscillation was better than the chaos behaviour beyond the natural frequency, which did not occur as 
predicted by the traditional analysis framework. In the frequency domain, intra-well oscillations, 1:3 subharmonic, inter-well oscillations appeared with increasing frequency in that order (see Fig. 23(b)).

Once $\mathrm{G}=0.21 \mathrm{~mm}$ was applied in case (a), four complete SMR cycles appeared in the frequency interval $[7.273 \mathrm{~Hz}, 7.385 \mathrm{~Hz}]$, which is marked by two black diamond points. The first snap-through motion and last jump-back motion of NES define the interval of SMR in Fig. 23(b). For the left boundary, at $7.273 \mathrm{~Hz}$, the LO always had the maximal amplitude. For the right boundary, at $7.385 \mathrm{~Hz}$, the LO possessed minimal local amplitude after several cycles of SMR. This indicates the effect of absorbing the energy of the SMR. The chaotic motion occupied two adjacent efficient TET, which resulted in the augmentation of LO amplitude in Fig. 23(a).

The SMR interval expanded to $[7.217 \mathrm{~Hz}, 7.47 \mathrm{~Hz}]$ under greater excitation, $\mathrm{G}=0.25 \mathrm{~mm}$. If $\mathrm{G}$ continued to increase to $0.32 \mathrm{~mm}$, the interval became broader $[7.15 \mathrm{~Hz}, 7.475 \mathrm{~Hz}]$. As $\mathrm{G}$ increased from 0.21 to $0.32 \mathrm{~mm}$, the left boundary, where SMR appeared, decreased from $7.273 \mathrm{~Hz}$ to $7.15 \mathrm{~Hz}$, while the right boundary, where SMR vanished, expanded from $7.385 \mathrm{~Hz}$ to $7.475 \mathrm{~Hz}$ accordingly. This demonstrates a broader efficient range for performing TET for a higher energy input before the resonance peak occurs.

Fig. 23(b) shows slight differences between the SMR near the left boundary and the SMR near the right boundary. In the low-frequency region, a single SMR cycle persists for a longer period than in the high-frequency region in Fig. 23(b). A shorter energy pumping time with a higher frequency excitation causes a faster decline in the amplitude of LO. Compared to the high-frequency band, a complete low-frequency SMR cycle consists of a more rapid amplitude growth process and a slower amplitude reduction. The high-frequency band SMR has the reverse characteristic in Fig. 23(a). A similar effect was found in the subsequent experimental results.

In $\mathrm{G}=0.36 \mathrm{~mm}$, the duration of amplitude decline of SMR has extended irregularly and caused a potential risk region near the left interval boundary. Meanwhile, the frequency range of SMR has achieved the maximum of [7.132 Hz, 7.549 Hz]. The case (a) design has the best robustness facing the uncertainty of excitation frequency under $\mathrm{G}=0.36 \mathrm{~mm}$. The $1: 3$ subharmonic oscillation appears in the low-frequency region. Then the SMR occurs in the vicinity of natural frequency. The systems return to a stable response if frequency continues to increase.

When $\mathrm{G}=0.4 \mathrm{~mm}$, the resonance peak appeared between $7.055 \mathrm{~Hz}$ and $7.25 \mathrm{~Hz}$. Within the resonance interval, the LO amplitude significantly exceeded the other cases, the NES lost its ability to absorb energy, and the system was at risk. A resonance peak occurred due to the existence of three solutions in the singularity equation in the low-frequency region, one of which had a large stable amplitude. However, in the vicinity of the natural frequency, $7.26 \mathrm{~Hz}$, the LO had a stable minimal amplitude of $2.8 \mathrm{~mm}$, which was located at the singularity point of the right SIM branch. So there is a trade-off relationship between the co-existence of the best performance of NES and the worst resonance peak at the amplitude of $\mathrm{G}=0.4 \mathrm{~mm}$ for case (a) design. The 
(a)

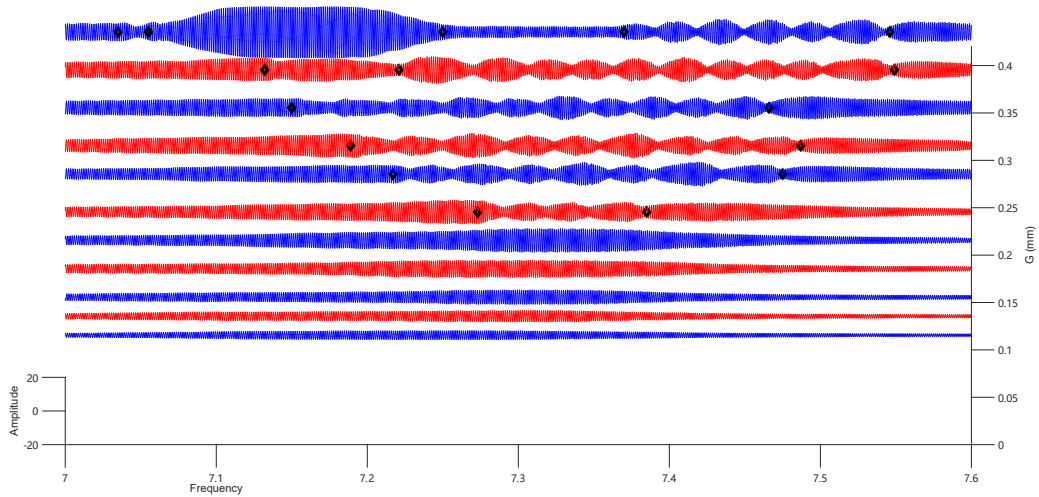

(b)

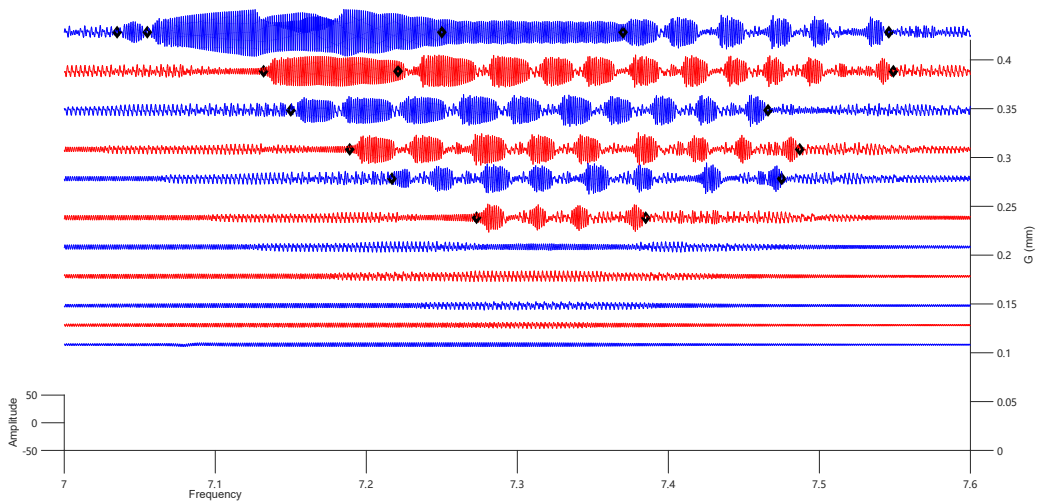

Fig. 23 Frequency response of LO (a) and NES (b) for case a. The amplitudes of excitation are selected as $0.08 \mathrm{~mm}, 0.10 \mathrm{~mm}, 0.12 \mathrm{~mm}, 0.15 \mathrm{~mm}, 0.18 \mathrm{~mm}, 0.21 \mathrm{~mm}, 0.25 \mathrm{~mm}, 0.28$ $\mathrm{mm}, 0.32 \mathrm{~mm}, 0.36 \mathrm{~mm}, 0.4 \mathrm{~mm}$. The black diamond distinguishes the SMR region from the resonance peak region.

best design also provided a possibility of worse behaviour at low frequency. So the feasibility of optimal design case (a) depended on the perturbation of harmonic force frequency. On the left side of the resonance peak region $[7.035 \mathrm{~Hz}$, $7.055 \mathrm{~Hz}]$, the NES also provided a small range of SMR. A small region of SMR appeared at low frequency. Then the resonance peak disrupted the optimal efficiency as frequency increased. Near the natural frequency, the system possessed an optimal steady state. Before the system returned to a stable response again, there was a broader range of SMR with increasing frequency. 
6.2 Case (b) experiment

With the pre-compression length increasing to $17.5 \mathrm{~mm}$, case (b) possessed larger negative and cubic stiffness values. Similarly to case (a), the system (b) was also used with 12 sets of sweeping frequency excitations of different amplitudes: $0.08 \mathrm{~mm}, 0.10 \mathrm{~mm}, 0.12 \mathrm{~mm}, 0.15 \mathrm{~mm}, 0.18 \mathrm{~mm}, 0.21 \mathrm{~mm}, 0.25$ $\mathrm{mm}, 0.28 \mathrm{~mm}, 0.32 \mathrm{~mm}, 0.36 \mathrm{~mm}, 0.40 \mathrm{~mm}, 0.44 \mathrm{~mm}$. Frequency varied from $7 \mathrm{~Hz}$ to $7.6 \mathrm{~Hz}$.

An essential characteristic of case (b) was the extensive range of apparent chaotic motion, which replaced the subharmonic motion of case (a). A larger depth value of potential well $\delta^{2} / 4 K$ enhanced the stability of intra-well oscillation. So the chaos motion occurred first for $\mathrm{G}=0.15 \mathrm{~mm}$. Meanwhile, the inter-well subharmonic motion appeared at a lower amplitude, $\mathrm{G}=0.1 \mathrm{~mm}$. A single and weak SMR was also observed for $\mathrm{G}=0.18 \mathrm{~mm}$, which is lower than the SMR occurrence threshold of case $(\mathrm{a})(\mathrm{G}=0.21 \mathrm{~mm})$ in Fig. 24. The SMR was generated near the natural frequency and divided the chaotic region. The chaos region moved to both lower and higher frequency sides as the excitation increased.

For excitation $\mathrm{G}$ from $0.21 \mathrm{~mm}$ to $0.32 \mathrm{~mm}$, the frequency interval for SMR occurrence expanded from a narrow range $[7.296 \mathrm{~Hz}, 7.314 \mathrm{~Hz}]$ to $[7.18 \mathrm{~Hz}$, $7.475 \mathrm{~Hz}$ ]. When $\mathrm{G}$ became $0.36 \mathrm{~mm}$, a potential resonance peak also occurred. However, the frequency range it occupied $[7.131 \mathrm{~Hz}, 7.18 \mathrm{~Hz}]$ was narrower than that of case (a). This tendency was more obvious in the response of NES under $\mathrm{G}=0.4 \mathrm{~mm}$, where the reasons peak region was $[7.085 \mathrm{~Hz}, 7.235 \mathrm{~Hz}]$. This range was far narrower than the $[7.05 \mathrm{~Hz}, 7.549 \mathrm{~Hz}]$ of case (a). The resonance peak occurred when the system did not give the stable response at $7.26 \mathrm{~Hz}$. At the same time, the interval in which SMR occurred widened to [7.235 Hz, $7.564 \mathrm{~Hz}]$.

At the amplitude $\mathrm{G}=0.44 \mathrm{~mm}$, the first signs of the steady-state response of LO at the natural frequency of $7.25 \mathrm{~Hz}$ appeared. SMR range still dominated the extensive range $[7.259 \mathrm{~Hz}, 7.587 \mathrm{~Hz}]$ and moved to a higher frequency side. An effective SMR range was also enhanced in a high energy input case. A larger compression length can reinforce the amplitude threshold required for the emergence of a steady state (saturation of absorbing energy).

\subsection{Case (c) experiment}

Case (c) could be achieved by continuing to compress the pre-compression length of $21 \mathrm{~mm}$. Case (c) was used with the same amplitude condition as case (b), except for the $0.44 \mathrm{~mm}$. The negative stiffness continued to be enhanced.

Neither chaotic motion nor subharmonic motion is observed in Fig. 25. It can be interpreted as a model of strong bistable NES that the narrow distance between trigger line B and singularity line D causes the phase trajectory to start snap-through motion and jump to the right branch of the SIM as soon 
(a)

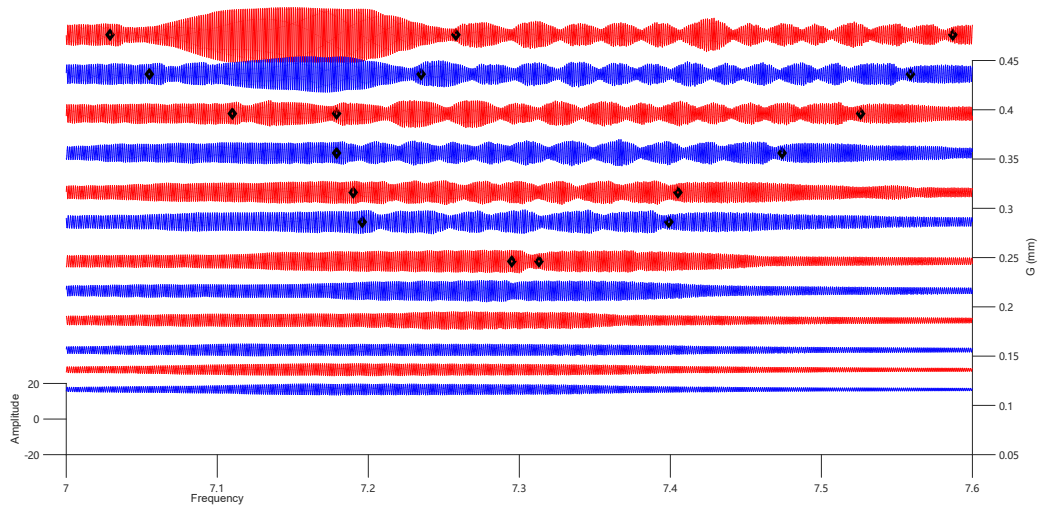

(b)

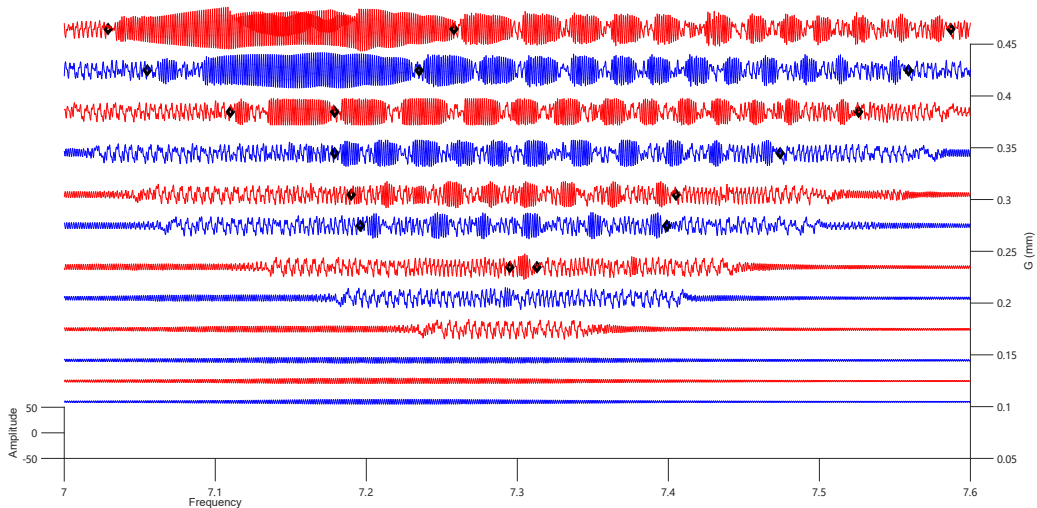

Fig. 24 Frequency response of LO (a) and NES (b) for case b. The amplitudes of excitation are selected as $0.08 \mathrm{~mm}, 0.10 \mathrm{~mm}, 0.12 \mathrm{~mm}, 0.15 \mathrm{~mm}, 0.18 \mathrm{~mm}, 0.21 \mathrm{~mm}, 0.25 \mathrm{~mm}, 0.28 \mathrm{~mm}$, $0.32 \mathrm{~mm}, 0.36 \mathrm{~mm}, 0.40 \mathrm{~mm}, 0.44 \mathrm{~mm}$. The black diamond distinguishes the SMR region from the resonance peak region.

as it comes out of the well. So, before the system oscillates around the right branch of the SIM, the chaos motion is replaced by an intra-well oscillation.

This first SMR appears at $\mathrm{G}=0.18 \mathrm{~mm}$, which is the same as case (b) in Fig. 25. The frequency range increases from $[7.189 \mathrm{~Hz}, 7.20 \mathrm{~Hz}]$ to $[7.051 \mathrm{~Hz}$, $7.417 \mathrm{~Hz}]$, as the excitation amplitude rises from $0.18 \mathrm{~mm}$ to $0.36 \mathrm{~mm}$. The SMR range shifts to a lower frequency side.

The range of potential resonance peak is $[7.075 \mathrm{~Hz}, 7.19 \mathrm{~Hz}]$ for $\mathrm{G}=0.4 \mathrm{~mm}$. Compared with the resonance peak in case (b) for $\mathrm{G}=0.36 \mathrm{~mm}$, the threshold for the occurrence of resonance increases and its appearance is delayed. The resonance situation is improved. 
(a)

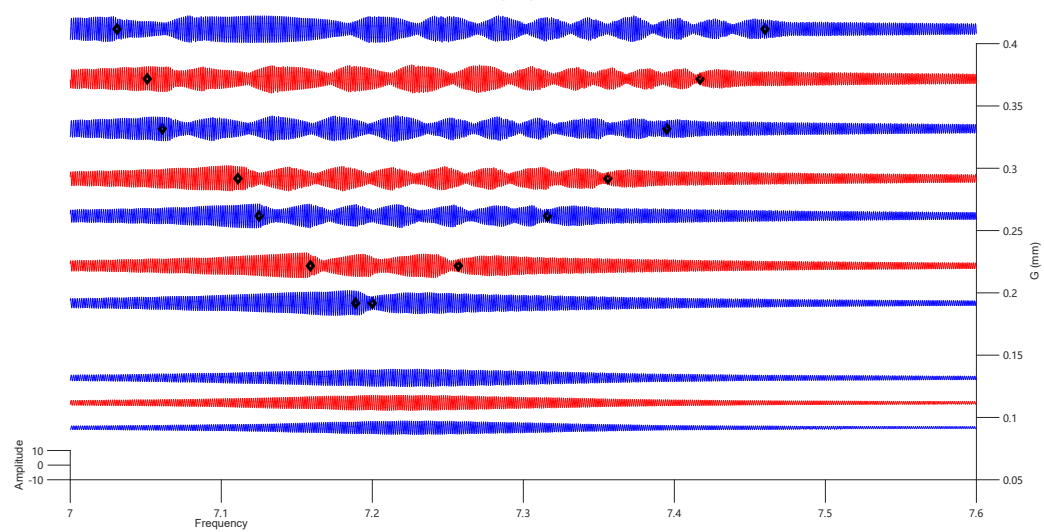

(b)

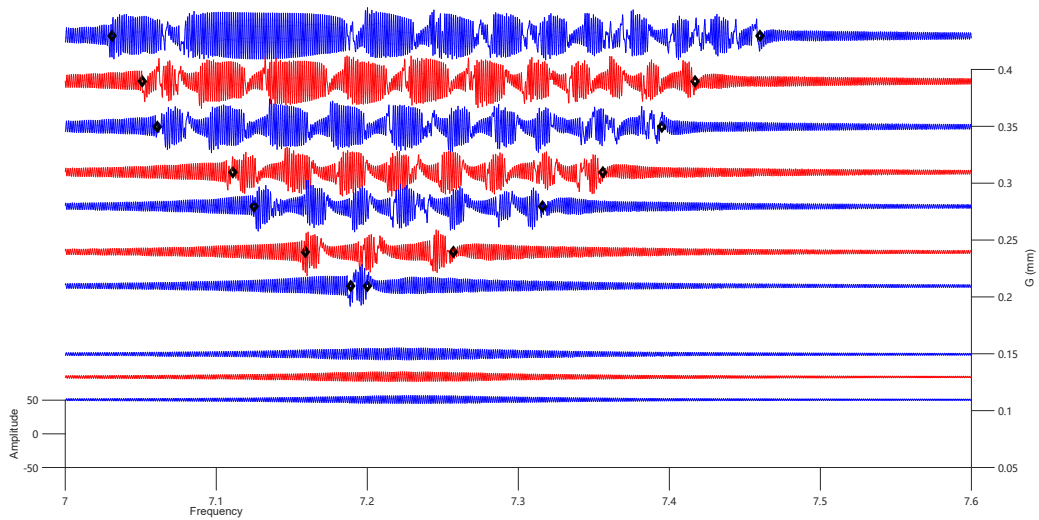

Fig. 25 Frequency response of LO (a) and NES (b) for case-c. The amplitude of excitation are selected as $0.08 \mathrm{~mm}, 0.10 \mathrm{~mm}, 0.12 \mathrm{~mm}, 0.18 \mathrm{~mm}, 0.21 \mathrm{~mm}, 0.25 \mathrm{~mm}, 0.28 \mathrm{~mm}, 0.32 \mathrm{~mm}$, $0.36 \mathrm{~mm}, 0.40 \mathrm{~mm}$. The black diamond distinguishes the SMR region and resonance peak region

\section{Conclusion}

The study focuses on the qualitative analysis of response regimes in bistable NES. Several main conclusions can be drawn:

1. When the adapted complex variable method, which defines the equilibrium point as an original coordinate, is performed to better approach the dynamic behaviours of intra-well oscillation, the numerical investigation reveals its natural stability of intra-well oscillation. This method gives a good fitting result and has been compared with the numerical results in the frequency domain. The actual phase trajectory of intra-well oscillates along with the constructed local SIM, which describes the low energy behaviours better than the classic method. But the local SIM's reliability is constrained to small energy levels. 
2. A simplified model of triggering chaos has proposed that the phase trajectory expands in a circular form with the equilibrium point as the centre within the pseudo-separatrix. Despite being simple, the chosen approach enabled us to predict the analytical harmonic excitation at which chaos occurred. The numerical chaos boundary has proved the reliability of its analytical prediction in weak negative stiffness cases.

3. The relative position between the chaos trigger line and the SIM structure has been proposed to illustrate the variation of the triggering SMR condition. The variety in relative position of those lines enables us to explain the alternation of stage in various negative stiffness cases and for various energy levels. On the other hand, the location of chaos trigger line B in the SIM structure classifies the bistable NES as a weak, modest, strong or abnormal bistable NES. A more efficient way to dissipate energy has been found in the modest bistable case, due to the small distance between the chaos trigger line and the singularity line.

4. The frequency-response experiment on LO amplitude was carried out to validate the feasibility of the adapted variables complex method. Good agreement between the theoretical and experimental results of intra-well oscillation under different negative stiffnesses was observed. The variety of characteristic responses in 3 different negative stiffness cases has been revealed experimentally. Optimal design of a modest bistable NES provides the broadest frequency range of SMR for the same excitation input and helps to reduce the risk of resonance peak.

\section{Compliance with Ethical Standards}

Conflict of Interest: The authors declare that they have no conflict of interest.

Funding: The authors acknowledge the Chinese Scholarship Council under Grant No. 201801810128 for their financial support.

Data Availability Statements: The authors declare that the data supporting the findings of this study are available within the article.

\section{References}

1. Vakakis, A.F., Gendelman, O.V., Bergman, L.A., McFar-land, M.D., Kerschen, G., Lee, Y.S.: Nonlinear Targeted Energy Transfer in Mechanical and Structural Systems, Springer, Amsterdam (2009)

2. Vakakis, A. F., Gendelman, O.v.: Energy pumping in nonlinear mechanical oscillators: partii-resonance capture, J.Appl Mech.,68(1), 42-48(2001)

3. Gendelman, O., Manevitch, L., Vakakis, A., and M'Closkey, R.: Energy Pumping in Nonlinear Mechanical Oscillators: Part I - Dynamics of the Under-lying Hamiltonian Systems, ASME J. Appl. Mech.,68(1), pp. 34-41(2001)

4. Starosvetsky. Y., Gendelman, O.V.: Strongly modulated response in forced 2DOF oscillatory system with essential mass and potential asymmetry, Physica D: Nonlinear Phenomena, 237(13), 1719-1733(2008) 
5. Starosvetsky,Y., and Gendelman,O.: Responseregimes of linear oscillator coupled to nonlinear energysink with harmonic forcing and frequency detuning, J.Sound Vib., 315(3), 746-765(2008)

6. Starosvetsky Y, Gendelman O.: Attractors of harmonically forced linear oscillator with attached nonlinear energy sink. ii: Optimization of a nonlinearvibration absorber, Nonlinear Dyn., 51(1-2), 47-57(2008)

7. Vaurigaud B, Savadkoohi A T, Lamarque C H.: Targeted energy transfer with parallel nonlinear energy sinks. part i: design theory and numericalresults, Nonlinear Dyn., 66(4), 763-780(2011).

8. McFarland D M, Kerschen G, Kowtko J J, et al.: Experimental investigation of targeted energy transfers instrongly and nonlinearly coupled oscillators, J. Acoust. Soc. Am., 118(2), 791-799(2005)

9. Bellet R, Cochelin B, Herzog P, et al.: Experimental study of targeted energy transferfrom an acoustic system to a nonlinear membrane absorber, J.Sound Vib., 329(14), 2768-2791(2020)

10. AL-Shudeifat M A.: Nonlinear energy sinks with piecewise-linear nonlinearities, Int.J Non Linear Mech, 14(12),124501(2019)

11. Saeed A S, AL-Shudeifat M A, Vakakis A F.: Rotary-oscillatory nonlinear energy sink of robust performanc, Int.J Non Linear. Mech, 117, 103249(2019)

12. Li T, Gourc E, Seguy S, et al.: Dynamics of two vibro-impact nonlinear energy sinks in parallel under periodic and transient excitations. Int.J Non Linear. Mech, 90, $100-$ 110(2017)

13. D. Qiu, S. Seguy, M. Paredes.: Design criteria for optimally tuned vibro-impact nonlinear energy sink, J. Sound Vib, 442, 497-513(2019).

14. Qiu D, Seguy S, Paredes M.: Tuned nonlinear energy sink with conical spring: design theory and sensitivity analysis, J.Mech.Design, 140(1), 011404(2018)

15. Al-Shudeifat, Mohammad A.: Highly efficient nonlinear energy sink. Nonlinear Dyn, 76(4), 1905-1920(2014)

16. Al-Shudeifat M A, Wierschem N, Quinn D D, et al.: Numerical and experimental investigation of a highly effective single-sided vibro-impact non-linear energy sink for shock mitigation. Int J Non Linear Mech, 52, 96-109(2013).

17. Chen Y Y, Qian Z C, Zhao W, et al., A magnetic bi-stable nonlinear energysink for structural seismic control, J. Sound Vib.,473,115233(2020)

18. Gourc E, Seguy S, Michon G, et al.: Quenching chatter instability in turning process with a vibro-impact nonlinear energy sink, J.Sound Vib., 355, 392-406(2015)

19. Manevitch L I.: The description of localized normal modes in a chain of nonlinear coupled oscillators using complex variables. Nonlinear Dyn, 25(1),95-109(2001)

20. Manevitch L I, Sigalov G, Romeo F, et al.: Dynamics of a linear oscillator coupled to a bistable light attachment: analytical study, J. Appl. Mech., 81(4), 041011(2014)

21. Romeo F, Sigalov G, Bergman L A, et al.: Dynamics of a linear oscillator coupled to a bistable light attachment: numerical study, J. Comput. Nonlinear. Dynam, 10(1), $011007(2015)$

22. Bitar D, Savadkoohi A T, Lamarque C H, et al.: Extended complexification method to study nonlinear passive control. Nonlinear Dyn, 99(2), 1433-1450(2020).

23. Qiu D, Li T, Seguy S, et al.: Efficient targeted energy transfer of bistable nonlinear energy sink: application to optimal design, Nonlinear Dyn, 92(2), 443-461(2018)

24. D. Qiu, M. Paredes, S. Seguy.: Variable pitch spring for nonlinear energy sink: application to passive vibration control, Proc Inst Mech Eng C J Mech Eng Sci, 233, 611$622(2019)$.

25. Z. Wu, S. Seguy, M. Paredes.: Basic Constraints for Design Optimization of Cubic and Bistable NES[J]. J. Vib. Acoust, 144, 1-51(2021).

26. Fang X, Wen J, Yin J, et al.: Highly efficient continuous bistable nonlinear energy sink composed of a cantilever beam with partial constrained layer damping, Nonlinear Dyn, 87(4), 2677-2695(2017)

27. Benacchio S, Malher A, Boisson J, et al.: Design of a magnetic vibration absorber with tunable stiffnesses, Nonlinear Dyn, 85(2), 893-911(2016)

28. Pennisi G, Mann B P, Naclerio N, et al.: Design and experimental study of a nonlinear energy sink coupled to an electromagnetic energy harvester, J.Sound Vib, 437, $340-357(2018)$ 
29. Pirrera A, Avitabile D, Weaver P M.: Bistable plates for morphing structures: a refined analytical approach with high-order polynomials, Int J.Solids Struct, 47(25-26), 3412$3425(2010)$

30. Johnson D R, Thota M, Semperlotti F, et al. On achieving high and adaptable damping via a bistable oscillator, Smart Mater Struct, 22(11), 115027(2013)

31. Iurasov V, Mattei P O.: Bistable nonlinear damper based on a buckled beam configuration, Nonlinear Dyn, 99(3), 1801-1822(2020)

32. Romeo F, Manevitch L I, Bergman L A, et al.: Transient and chaotic low-energy transfers in a system with bistable nonlinearity, Chaos, 25(5), 053109(2015)

33. Dekemele K, Van Torre P, Loccufier M.: Performance and tuning of a chaotic bi-stable NES to mitigate transient vibrations, Nonlinear Dyn, 98(3), 1831-1851(2019)

34. Farshidianfar A, Saghafi A.: Global bifurcation and chaos analysis in nonlinear vibration of spur gear systems, Nonlinear Dyn, 75(4), 783-806(2014) 